Itai Shafrir · Gershon Wolansky

\title{
Moser-Trudinger and logarithmic HLS inequalities for systems
}

Received December 27, 2004

Abstract. We prove several optimal Moser-Trudinger and logarithmic Hardy-Littlewood-Sobolev inequalities for systems in two dimensions. These include inequalities on the sphere $S^{2}$, on a bounded domain $\Omega \subset \mathbb{R}^{2}$ and on all of $\mathbb{R}^{2}$. In some cases we also address the question of existence of minimizers.

\section{Introduction}

The Moser-Trudinger (MT) inequality (see [13]) on the two-sphere $S^{2}$ reads:

$f^{M}(u):=\int_{S^{2}} \frac{1}{2}|\nabla u|^{2} d \omega-M \log \left(\int_{S^{2}} e^{u} \frac{d \omega}{4 \pi}\right) \geq-C, \quad \forall u \in H^{1}\left(S^{2}\right), \int_{S^{2}} u d \omega=0$,

where $0<M \leq 8 \pi$. The MT inequality plays an important role in problems of prescribing Gauss curvature (see Aubin [1], Chang-Yang [5] and the references therein). A sharp version of [1.1], which is due to Onofri [15], states that (1.1) is valid with the optimal $C=0$ on the right hand side. Moser's original proof of 1.1 was obtained as a corollary of his stronger inequality:

$$
\int_{S^{2}} e^{4 \pi u^{2}} \leq C, \quad \forall u \in H^{1}\left(S^{2}\right) \text { with } \int_{S^{2}} u=0 \text { and } \int_{S^{2}}|\nabla u|^{2}=1 .
$$

Onofri's proof of the sharp form of [1.1), i.e. with $C=0$, used estimates of Aubin [1] and the conformal invariance of the functional. Other proofs of Onofri's result were later given by Hong [10] and by Osgood, Phillips and Sarnak [16]. Of particular interest to us is the alternative derivation of Onofri's inequality by Beckner [3] (see also Carlen and Loss [4]), which is based on a duality principle and on Lieb's sharp form of the HardyLittlewood-Sobolev inequality ${ }_{1}^{1}$ On $S^{2}$ the dual inequality takes the form

$$
\psi_{S^{2}}(\rho):=\int_{S^{2}} \rho \log \rho+\frac{1}{4 \pi} \int_{S^{2}} \int_{S^{2}} \rho\left(\omega_{1}\right)\left(\log \left|\omega_{1}-\omega_{2}\right|\right) \rho\left(\omega_{2}\right) d \omega_{1} d \omega_{2} \geq-C
$$

I. Shafrir, G. Wolansky: Technion - Israel Institute of Technology, 32000 Haifa, Israel; e-mail: shafrir@math.technion.ac.il, gershonw@math.technion.ac.il

Mathematics Subject Classification (2000): Primary 35J60

1 Actually Beckner's result generalizes Onofri's inequality to any dimension. 
for all $\rho \in \Gamma_{M}\left(S^{2}\right)$ if $M \leq 8 \pi$, where

$$
\Gamma_{M}\left(S^{2}\right):=\left\{\rho \geq 0: \int_{S^{2}} \rho \log \rho<\infty \text { and } \int_{S^{2}} \rho=M\right\},
$$

and $\left|\omega_{1}-\omega_{2}\right|$ stands for the Euclidean distance between $\omega_{1}$ and $\omega_{2}$ in $\mathbb{R}^{3}$.

The generalization of the functional $\psi_{S^{2}}$ to the system case is the functional

$\Psi_{S^{2}}(\boldsymbol{\rho})=\sum_{i \in I} \int_{S^{2}} \rho_{i} \log \rho_{i}+\frac{1}{4 \pi} \sum_{i, j \in I} a_{i, j} \int_{S^{2}} \int_{S^{2}} \rho_{i}\left(\omega_{1}\right)\left(\log \left|\omega_{1}-\omega_{2}\right|\right) \rho_{j}\left(\omega_{2}\right) d \omega_{1} d \omega_{2}$,

considered on the domain

$$
\boldsymbol{\Gamma}_{\boldsymbol{M}}\left(S^{2}\right)=\left\{\boldsymbol{\rho}=\left(\rho_{i}\right)_{i \in I}: \rho_{i} \geq 0, \int_{S^{2}} \rho_{i} \log \rho_{i}<\infty \text { and } \int_{S^{2}} \rho_{i}=M_{i}, \forall i\right\},
$$

where $I:=\{1,2, \ldots, n\}$ and $A:=\left\{a_{i, j}\right\}$ is a symmetric $n$ by $n$ matrix. In what follows we assume that $a_{i, j} \geq 0$ for all $i, j$, but later we shall also study other classes of matrices. The duality relation between 1.1 and 1.3 can be extended, under the additional hypothesis that $A$ is positive definite, to a duality between $(1.5)$ and the functional

$$
F^{\boldsymbol{M}}(\boldsymbol{u})=\frac{1}{2} \sum_{i, j \in I} a_{i, j} \int_{S^{2}} \nabla u_{i} \nabla u_{j}-\sum_{i \in I} M_{i} \log \left(\frac{1}{4 \pi} \int_{S^{2}} \exp \left(\sum_{j \in I} a_{i, j} u_{j}\right)\right)
$$

over the class

$$
\mathcal{H}_{n}\left(S^{2}\right):=\left\{\boldsymbol{u} \in\left(H^{1}\left(S^{2}\right)\right)^{n}: \int_{S^{2}} u_{i}=0, \forall i\right\}
$$

Note that in the scalar case $n=1$ it follows from the MT inequality that a necessary and sufficient condition for the boundedness from below of $f^{M}$ and $\psi_{S^{2}}$ over $\mathcal{H}_{1}\left(S^{2}\right)$ and $\Gamma_{M}\left(S^{2}\right)$, respectively, is $M \leq 8 \pi$. The analogue of this condition to the system case turns out to be a set of $2^{n}-1$ inequalities involving the quadratic polynomials

$$
\Lambda_{J}(\boldsymbol{M})=8 \pi \sum_{i \in J} M_{i}-\sum_{i, j \in J} a_{i, j} M_{i} M_{j}=\sum_{i \in J} M_{i}\left(8 \pi-\sum_{j \in J} a_{i, j} M_{j}\right),
$$

for every nonempty subset $J \subseteq I$. The polynomial $\Lambda_{I}$ was first introduced by Chanillo and Kiessling [6] in their study of entire solutions of Liouville systems in $\mathbb{R}^{2}$. A set of conditions ("subcritical"),

$$
\Lambda_{J}(\boldsymbol{M})>0, \quad \forall J \subseteq I, J \neq \emptyset,
$$

was used in [7] for the study of a related variational problem on bounded domains in $\mathbb{R}^{2}$ and the associated minimizers (see also below). On the other hand, a simple rescaling argument (as in [7, Lemma 2.2]) shows that if for some $J, \Lambda_{J}(\boldsymbol{M})<0$, then $F^{\boldsymbol{M}}$ and $\Psi_{S^{2}}$ are unbounded from below. Wang [20] proved an analogous result to that of [7] for compact surfaces, showing that the subcritical condition (1.9) is sufficient for the boundedness of (the analogue of) $F^{M}$ in this case. A natural question that we address 
here is whether the bound still holds in the critical case, i.e. when we turn some, or all, of the inequalities in (1.9) into equalities to get the weaker condition

$$
\Lambda_{J}(\boldsymbol{M}) \geq 0, \quad \forall J \subseteq I, J \neq \emptyset .
$$

One of our main results, Theorem 2 , asserts that the bound for $\Psi_{S^{2}}$ (res. $F^{\boldsymbol{M}}$ ) indeed holds under assumption (1.10) if $a_{i, i}>0$ for all $i$, but a slightly stronger condition is needed if we allow zero diagonal elements (see 2.16 below). So far, results for the critical case were obtained only for very special systems by Wang [20] and by Jost and Wang [11] (see (2.11) and below). We shall also consider analogous functionals on the whole plane $\mathbb{R}^{2}$ as well as on bounded domains $\Omega \subset \mathbb{R}^{2}$. In certain cases, when the bound is verified, we shall address the question of the existence of minimizers.

In the next section we introduce the full details of our main results. Some of them were announced in [18]. The proofs are given in the following sections.

\section{Description of main results}

Before stating our main results we want to focus on two important notions which demonstrate the similarities and differences between the scalar problem and the vectorial one. The first notion is duality.

In the scalar case $n=1$, the functionals 1.1 on $\mathcal{H}_{1}\left(S^{2}\right)$ and 1.3 on $\Gamma_{M}\left(S^{2}\right)$ are dual in the sense that both are bounded or unbounded from below, simultaneously. Moreover, a minimizer $u$ for $f^{M}$, if exists, is related to a minimizer $\rho$ of $\psi_{S^{2}}$ via $\rho=M e^{u} / \int_{S^{2}} e^{u}$ and $-\Delta u=\rho-M / 4 \pi$. This duality extends to the vector functionals $\Psi_{S^{2}}$ (on $\boldsymbol{\Gamma}_{M}\left(S^{2}\right)$ ) and $F^{\boldsymbol{M}}$ (on $\mathcal{H}_{n}\left(S^{2}\right)$ ) provided the matrix $\left\{a_{i, j}\right\}$ is positive definite (see Section 3 . However, our results for $\Psi_{S^{2}}$ do not require this condition. Our basic assumption is

$$
a_{i, j} \geq 0, \quad \forall i, j
$$

but we shall also study other classes of matrices in Subsection 5.2

Next we turn to the notion of conformal invariance. It is known that $\psi_{S^{2}}(\rho)$ is conformally invariant in the critical case $M=8 \pi$. By this we mean that the 1.h.s. of 1.3 is invariant under the conformal action,

$$
\rho \mapsto \rho^{\tau}:=(\rho \circ \tau) \cdot\left|\mathcal{J}_{\tau}\right|
$$

where $\tau$ is any conformal automorphism of $S^{2}$ and $\mathcal{J}_{\tau}$ is its Jacobian (here and in the following we do not distinguish between conformal and anti-conformal automorphisms). We shall often apply 2.2 for a special class of automorphisms, $\left\{\tau_{y, \alpha}: y \in S^{2}, \alpha \in \mathbb{R}_{+}\right\}$, defined as follows:

$$
\tau_{y, \alpha}(x)=\mathcal{S}_{y}^{-1}\left(\alpha \mathcal{S}_{y}(x)\right), \quad \forall x \in S^{2},
$$

where $\mathcal{S}_{y}$ is the stereographic projection satisfying $\mathcal{S}_{y}(y)=\infty$. However, in the vectorial case (i.e. $n \geq 2$ ) it turns out that the condition analogous to $M=8 \pi$, namely $\Lambda_{I}(\boldsymbol{M})=0$, is not sufficient, in general, to ensure the conformal invariance of $\Psi_{S^{2}}$ on $\Gamma_{M}\left(S^{2}\right)$. 
In fact, $\Psi_{S^{2}}$ is clearly invariant with respect to the action 2.2 (applied to $\rho=\rho_{i}$, $i \in I$ ) when $\tau$ is an isometry of $S^{2}$. But the conformal group also contains other automorphisms whose action is more transparent when we use the stereographic projection to transform the problem to $\mathbb{R}^{2}$. Using $\mathcal{S}=\mathcal{S}_{\mathcal{N}}(\mathcal{N}$ denoting the north pole $)$ we associate to each $\rho: S^{2} \rightarrow \mathbb{R}^{n}$ a function $\tilde{\rho}: \mathbb{R}^{2} \rightarrow \mathbb{R}^{n}$ via the transformation (see [3, 4])

$$
\left\{\begin{array}{l}
\tilde{\rho}_{i} \leftrightarrow \rho_{i}=\left[\tilde{\rho}_{i} \cdot\left(1+|x|^{2}\right)^{2} / 4\right] \circ \mathcal{S}, \\
\rho_{i} \leftrightarrow \tilde{\rho}_{i}=\frac{4}{\left(1+|x|^{2}\right)^{2}} \cdot\left(\rho_{i} \circ \mathcal{S}^{-1}\right), \quad \forall i \in I .
\end{array}\right.
$$

Note that

$$
\left|\mathcal{S}^{-1}(x)-\mathcal{S}^{-1}(y)\right|=\frac{2|x-y|}{\left(1+|x|^{2}\right)^{1 / 2}\left(1+|y|^{2}\right)^{1 / 2}}, \quad \forall x, y \in \mathbb{R}^{2} .
$$

By a simple computation, using (2.5), we obtain for $\rho$ and $\tilde{\rho}$ which are related by (2.4),

$$
\begin{aligned}
\Psi_{S^{2}}(\boldsymbol{\rho})=\widetilde{\Psi}_{\mathbb{R}^{2}}(\tilde{\boldsymbol{\rho}}):= & \sum_{i \in I} \int_{\mathbb{R}^{2}} \tilde{\rho}_{i} \log \tilde{\rho}_{i} d x \\
& +\frac{1}{4 \pi} \sum_{i, j \in I} a_{i, j} \int_{\mathbb{R}^{2}} \int_{\mathbb{R}^{2}} \tilde{\rho}_{i}(x)(\log |x-y|) \tilde{\rho}_{j}(y) d x d y \\
& +\sum_{i \in I} v_{i} \int_{\mathbb{R}^{2}} \tilde{\rho}_{i} \log \left(1+|x|^{2}\right) d x-\frac{1}{4 \pi} \Lambda_{I}(\boldsymbol{M}) \log 2,
\end{aligned}
$$

with

$$
v_{i}=2-\frac{1}{4 \pi} \sum_{j \in I} a_{i, j} M_{j}, \quad \forall i \in I
$$

It is clear that

$$
\int_{\mathbb{R}^{2}} \tilde{\rho}_{i}=\int_{S^{2}} \rho_{i}, \quad \forall i \in I .
$$

Moreover, using the arguments of [4] it can be shown that $\Psi_{S^{2}}$ is bounded below over $\boldsymbol{\Gamma}_{\boldsymbol{M}}\left(S^{2}\right)$ (see $(1.6)$ ) if and only if $\widetilde{\Psi}_{\mathbb{R}^{2}}$ is bounded below on

$$
\begin{aligned}
\boldsymbol{\Gamma}_{\boldsymbol{M}}\left(\mathbb{R}^{2}\right)=\left\{\tilde{\boldsymbol{\rho}}: \tilde{\rho}_{i} \geq 0,\right. & \int_{\mathbb{R}^{2}} \tilde{\rho}_{i}\left|\log \tilde{\rho}_{i}\right|<\infty, \\
& \left.\int_{\mathbb{R}^{2}} \tilde{\rho}_{i}=M_{i}, \int_{\mathbb{R}^{2}} \tilde{\rho}_{i} \log \left(1+|x|^{2}\right)<\infty, \forall i \in I\right\} .
\end{aligned}
$$

Now we can observe that the functional

$$
\Psi_{\mathbb{R}^{2}}(\tilde{\boldsymbol{\rho}})=\sum_{i \in I} \int_{\mathbb{R}^{2}} \tilde{\rho}_{i} \log \tilde{\rho}_{i} d x+\frac{1}{4 \pi} \sum_{i, j \in I} a_{i, j} \int_{\mathbb{R}^{2}} \int_{\mathbb{R}^{2}} \tilde{\rho}_{i}(x)(\log |x-y|) \tilde{\rho}_{j}(y) d x d y
$$


is invariant with respect to translations, i.e. $\tilde{\rho}_{i}(x) \mapsto \tilde{\rho}_{i}(x+v)$ for all $i \in I$ (for any fixed $v \in \mathbb{R}^{2}$ ), and dilatations, i.e. $\tilde{\rho}_{i}(x) \mapsto \alpha^{2} \tilde{\rho}_{i}(\alpha x)$ for all $i \in I$ (for any fixed $\alpha>0$ ), provided that $\Lambda_{I}(\boldsymbol{M})=0$. But clearly the difference between the two functionals,

$$
\widetilde{\Psi}_{\mathbb{R}^{2}}(\tilde{\boldsymbol{\rho}})-\Psi_{\mathbb{R}^{2}}(\tilde{\boldsymbol{\rho}})=\sum_{i \in I} v_{i} \int_{\mathbb{R}^{2}} \tilde{\rho}_{i} \log \frac{1+|x|^{2}}{2} d x,
$$

is not invariant with respect to translations and dilatations unless $v_{i}=0$ for all $i \in I$. We shall call this last case, i.e. when

$$
\sum_{j \in I} a_{i, j} M_{j}=8 \pi, \quad \forall i \in I,
$$

the conformal case. Indeed, from the above we see that 2.11 is a necessary and sufficient condition for the full invariance of the functional $\Psi_{S^{2}}$ with respect to the conformal group of the sphere. Evidently, (2.11) implies that $\Lambda_{I}(\boldsymbol{M})=0$, but as explained above, the converse is false in general. We should mention that Wang [20] studied a special case of the conformal case (2.11) in which the positive definite matrix $A$ is stochastic, that is,

$$
\sum_{i \in I} a_{i, j}=1, \quad \forall j \in I,
$$

and the vector of masses $\boldsymbol{M}$ satisfies $M_{i}=8 \pi$ for all $i \in I$. Under these assumptions he proved that the functional $F^{\boldsymbol{M}}$ is bounded below on $\mathcal{H}_{n}\left(S^{2}\right)$ (this clearly implies boundedness also when $M_{i} \leq 8 \pi$ for all $i$ ). Actually, the result of Wang is more general since he studied a functional which is defined on any closed surface $\Sigma$ (i.e. two-dimensional compact Riemannian manifold without boundary). In Section 3 we shall prove the following optimal result for the conformal case on $S^{2}$, which can be viewed as the natural generalization of the results of Onofri and Beckner to the system case (since it gives the optimal additive constant). Here again we see the advantage of using the dual formulation: it allows us to deduce easily the system analogue from Beckner's scalar result.

We recall that a symmetric matrix $A$ is called irreducible if for all $i, j \in I$, there exist $\left\{k_{1}, \ldots, k_{l}\right\} \in I$ with $k_{1}=i$ and $k_{l}=j$ such that $a_{k_{1}, k_{2}} \cdot a_{k_{2}, k_{3}} \cdots a_{k_{l-1}, k_{l}} \neq 0$. Equivalently, $A$ is irreducible if there is no $\emptyset \neq J \varsubsetneqq I$ such that $a_{i, j}=0$ for all $i \in J$ and $j \notin J$. Any symmetric matrix $A$ can be decomposed into a sum of irreducible matrices, inducing a decomposition of the functional $\Psi_{S^{2}}$ into a sum of independent functionals, each corresponding to an irreducible factor. The assumption of irreducibility is useful for some uniqueness questions.

Theorem 1. Let $A$ be a symmetric matrix satisfying 2.1] and $\boldsymbol{M} \in \mathbb{R}_{+}^{n}$ such that 2.11] holds. Setting

$$
\boldsymbol{\rho}^{0}=\left(\frac{M_{1}}{4 \pi}, \ldots, \frac{M_{n}}{4 \pi}\right) \quad \text { and } \quad \tilde{\boldsymbol{\rho}}^{0}=\left(\frac{M_{1}}{\pi\left(1+|x|^{2}\right)^{2}}, \ldots, \frac{M_{n}}{\pi\left(1+|x|^{2}\right)^{2}}\right)
$$

we have: 
(i)

$$
\min _{\boldsymbol{\Gamma}_{\boldsymbol{M}}\left(S^{2}\right)} \Psi_{S^{2}}=\Psi_{S^{2}}\left(\boldsymbol{\rho}^{0}\right)
$$

and

$$
\min _{\Gamma_{M}\left(\mathbb{R}^{2}\right)} \Psi_{\mathbb{R}^{2}}=\Psi_{\mathbb{R}^{2}}\left(\tilde{\boldsymbol{\rho}}^{0}\right) .
$$

(ii) The conformal images (as defined in 2.2) of the constant vector $\boldsymbol{\rho}^{0}$ are minimizers in 2.13 .

(iii) The conformal images of $\tilde{\boldsymbol{\rho}}^{0}$ (i.e. $\left(\tilde{\boldsymbol{\rho}}^{0} \circ \tau\right) \cdot\left|\mathcal{J}_{\tau}\right|, \tau: \mathbb{R}^{2} \rightarrow \mathbb{R}^{2}$ conformal) are minimizers in (2.14).

(iv) If, in addition, the matrix $A$ is positive definite, then

$$
F^{M}(\boldsymbol{u}) \geq 0, \quad \forall \boldsymbol{u} \in \mathcal{H}_{n}\left(S^{2}\right)
$$

with equality if

$$
\boldsymbol{u}=\left(\frac{M_{1}}{8 \pi} \log \left|\mathcal{J}_{\tau}\right|, \ldots, \frac{M_{n}}{8 \pi} \log \left|\mathcal{J}_{\tau}\right|\right)+\boldsymbol{c},
$$

where $c$ is a constant vector.

(v) Assume now that A is irreducible. Then the minimizers given explicitly in (ii)-(iv) are the unique minimizers. Also, in the subconformal case, i.e. when $v_{i} \geq 0$ for all $i \in I$ with at least one strict inequality, $\rho^{0}$ is the unique minimizer in $(2.13)$, and if $A$ is positive definite, then $\boldsymbol{u}=\mathbf{0}$ is the unique minimizer in 2.15.

As explained above, for $n \geq 2$ the conformal case is exceptional among the critical configurations of $A$ and $\boldsymbol{M}$. Our next theorem provides an optimal criterion for boundedness from below of the functionals $\Psi_{S^{2}}(\rho)$ and $F^{\boldsymbol{M}}$ in the general case. It turns out that this criterion requires a slightly stronger condition than (1.10), namely,

$$
\left\{\begin{array}{l}
\Lambda_{J}(\boldsymbol{M}) \geq 0 \text { for all } \emptyset \neq J \subseteq I, \\
\text { if } \Lambda_{J}(\boldsymbol{M})=0 \text { for some } J, \text { then } a_{i, i}+\Lambda_{J \backslash\{i\}}(\boldsymbol{M})>0, \forall i \in J .
\end{array}\right.
$$

Remark 2.1. Note that 2.16 is equivalent to 1.10 if the matrix $A$ has a positive diagonal, that is, $a_{i, i}>0$ for all $i \in I$.

Theorem 2. Let $A$ be a symmetric matrix satisfying 2.1] and $\boldsymbol{M} \in \mathbb{R}_{+}^{n}$. Then:

(i) Condition 2.16 is necessary and sufficient for the boundedness from below of $\Psi_{S^{2}}$ on $\boldsymbol{\Gamma}_{\boldsymbol{M}}\left(S^{2}\right)$.

(ii) If, in addition, the matrix $A$ is positive definite, then condition 2.16 is necessary and sufficient for the boundedness from below of $F^{\boldsymbol{M}}$ on $\mathcal{H}_{n}\left(S^{2}\right)$.

Remark 2.2. In the general nonconformal critical case, in contrast with the conformal case, we do not know whether minimizers exist, both in (i) and (ii). What we do know is that $\rho^{0}=\left(M_{1} / 4 \pi, \ldots, M_{n} / 4 \pi\right)$ is not a minimizer for $\Psi_{S^{2}}$ and that $\boldsymbol{u}^{0} \equiv 0$ is not a minimizer for $F^{M}$ (although both are solutions of the corresponding Euler-Lagrange equations); see Proposition 3.1 below. In [19] we obtained a generalization of Theorem 2] i) for compact manifolds in dimension $N \geq 2$. 
So far we considered only nonnegative matrices $A$ (i.e. those satisfying (2.1)). However, there is interest in studying a more general class of systems, namely that of collaborating systems. These are systems associated with symmetric matrices $A$ which have the following structure: there exists a decomposition of $I$ as a disjoint union of $K(1 \leq K$ $\leq n$ ) subsets $I_{1}, \ldots, I_{K}$ such that

$$
\left\{\begin{array}{l}
a_{i, j} \geq 0, \quad \forall i, j \in I_{l}, l=1, \ldots, K \\
a_{i, j} \leq 0, \quad \forall i \in I_{l}, \forall j \in I_{m}, \forall l \neq m, 1 \leq l, m \leq K .
\end{array}\right.
$$

The case $K=1$ corresponds of course to a nonnegative matrix, but for $K \geq 2$, an assumption that we shall make in what follows, we obtain new types of matrices. Of particular interest is the extreme case $K=n$. Here all the $I_{l}$ 's are singletons and we get the condition

$$
a_{i, i} \geq 0, \forall i \quad \text { and } \quad a_{i, j} \leq 0, \forall i \neq j .
$$

In fact, Jost and Wang studied in [11] a special system of this type, the Toda system, which corresponds to the case where $A$ is the Cartan matrix for $S U(n+1)$, i.e.

$$
A=\left(\begin{array}{cccccc}
2 & -1 & 0 & \ldots & \ldots & 0 \\
-1 & 2 & -1 & \ddots & 0 & \vdots \\
0 & -1 & \ddots & \ddots & \ddots & \vdots \\
\vdots & \ddots & \ddots & \ddots & -1 & 0 \\
\vdots & 0 & \ddots & -1 & 2 & -1 \\
0 & \ldots & \ldots & 0 & -1 & 2
\end{array}\right)
$$

For $A$ in the class 2.18 the condition (1.10) (which is easily seen to be equivalent to 2.16 in this case) simplifies to

$$
0 \leq \Lambda_{\{i\}}(\boldsymbol{M})=8 \pi M_{i}-a_{i, i} M_{i}^{2}, \quad \forall i .
$$

Indeed, the validity of 1.10 for all singletons, $J=\{i\}$ for all $i$, implies its validity for all $J \subseteq I$, since all the off-diagonal elements of $A$ are nonpositive. In fact, in [11] it was proved that for the Toda system condition $(2.20)$, i.e. $M_{i} \leq 4 \pi$ for all $i$, is sufficient for the corresponding $F^{\boldsymbol{M}}$ to be bounded below on $\mathcal{H}_{n}\left(S^{2}\right)$ (necessity is known to hold true). Actually, Jost and Wang proved the analogue result on every 2-dimensional compact surface. Using the dual formulation we are able to obtain a very simple proof of the boundedness from below of $\Psi_{S^{2}}$ on $\boldsymbol{\Gamma}_{\boldsymbol{M}}\left(S^{2}\right)$ in this case. Moreover, we are able to compute the exact value of the infimum and to prove that the infimum is not achieved. We summarize our results for general collaborating systems in the following theorem.

Theorem 3. Let A be a symmetric matrix corresponding to a collaborating system with $K \geq 2$ blocks and let $\boldsymbol{M} \in \mathbb{R}_{+}^{n}$ be given. Then: 
(i) The validity of 2.16 for each $I_{l}$, i.e.

$$
\forall l=1, \ldots, K:\left\{\begin{array}{l}
\Lambda_{J}(\boldsymbol{M}) \geq 0 \text { for all } \emptyset \neq J \subseteq I_{l}, \text { and } \\
\text { if } \Lambda_{J}(\boldsymbol{M})=0 \text { for some } J \subseteq I_{l}, \\
\text { then } a_{i, i}+\Lambda_{J \backslash\{i\}}(\boldsymbol{M})>0, \forall i \in J,
\end{array}\right.
$$

is a necessary and sufficient condition for the boundedness from below of $\Psi_{S^{2}}$ on $\boldsymbol{\Gamma}_{\boldsymbol{M}}\left(S^{2}\right)$, and when $A$ is positive definite, for the boundedness from below of $F^{\boldsymbol{M}}$ on $\mathcal{H}_{n}\left(S^{2}\right)$.

(ii) If $K=n$, i.e. A satisfies 2.18, and if the critical case conditions, $a_{i, i} M_{i}=8 \pi$ for all $i$, are satisfied, then

$$
\inf _{\Gamma_{M}\left(S^{2}\right)} \Psi_{S^{2}}=\sum_{i \in I}\left[M_{i} \log \frac{M_{i}}{4 \pi}+\frac{M_{i}}{2 \pi} c_{0}\right]-\frac{1}{4 \pi} \sup _{\left(S^{2}\right)^{n}} W(\boldsymbol{x}),
$$

where

$$
W(\boldsymbol{x}):=\sum_{i \neq j}\left(-a_{i, j}\right) M_{i} M_{j} \log \left|x_{i}-x_{j}\right|
$$

and

$$
c_{0}=\int_{S^{2}} \log |x-y| d y=2 \pi(\log 4-1) .
$$

(iii) Under the assumptions of (ii), if we suppose in addition that A does not have a row of zeros (ignoring the diagonal), then the infimum in (2.22) is not attained. Moreover, any weak limit (in the sense of measures) of a minimizing sequence is of the form $\boldsymbol{\rho}=\left(M_{1} \delta_{x_{1}}, \ldots, M_{n} \delta_{x_{n}}\right)$, where $\boldsymbol{x}=\left(x_{1}, \ldots, x_{n}\right) \in\left(S^{2}\right)^{n}$ is a maximizer of $W$.

In Subsection 5.2 we shall present a variant of Theorem 3 (ii),(iii) for a more general class of matrices than (2.18).

Remark 2.3. Since the proof of part (i) of Theorem 3 uses only the scalar Moser-Trudinger inequality, which is known to be true on any two-dimensional compact surface (see $[9,[14]$ ), it follows that the assertion in (i) is valid in this more general setting (as proved in [11] for the Toda system).

Next we present two results on related variational problems, on $\mathbb{R}^{2}$ and on a bounded domain $\Omega \subset \mathbb{R}^{2}$. In the following we shall assume again that $A$ satisfies 2.1 . We shall first describe an entropy inequality which involves the functional $\Psi_{\mathbb{R}^{2}}$, already defined in 2.9. We claim that this functional is well defined on $\boldsymbol{\Gamma}_{\boldsymbol{M}}\left(\mathbb{R}^{2}\right)$ for every $\boldsymbol{M} \in \mathbb{R}_{+}^{n}$. Indeed, using (2.5), the stereographic projection and the obvious fact that the Euclidean distance between any two points on the unit sphere is less than or equal to 2, we get the elementary inequality

$$
\log |x-y| \leq \frac{1}{2} \log \left(1+|x|^{2}\right)+\frac{1}{2} \log \left(1+|y|^{2}\right), \quad \forall x, y \in \mathbb{R}^{2} .
$$


By (2.25) we obtain for any $\tilde{\boldsymbol{\rho}} \in \boldsymbol{\Gamma}_{\boldsymbol{M}}\left(\mathbb{R}^{2}\right)$, as in [4],

$$
\int_{\mathbb{R}^{2}} \int_{\mathbb{R}^{2}} \tilde{\rho}_{i}(x)(\log |x-y|) \tilde{\rho}_{j}(y) d x d y<\infty, \quad \forall i, j .
$$

On the other hand, using 3.3 below, we get

$$
\int_{\{|y-x| \leq 1\}} \tilde{\rho}_{i}(y) \log \left(\frac{1}{|x-y|}\right) d y \leq C, \quad \forall x \in \mathbb{R}^{2}, \forall i .
$$

Hence

$$
\int_{\mathbb{R}^{2}} \int_{\mathbb{R}^{2}} \tilde{\rho}_{i}(x)(\log |x-y|) \tilde{\rho}_{j}(y) d x d y>-\infty, \quad \forall i, j,
$$

and our claim follows. From the discussion after 2.8 it follows that $\Psi_{\mathbb{R}^{2}}$ is invariant with respect to translations and dilatations, but, in general, not with respect to the other conformal actions of $\mathbb{R}^{2}$. The invariance with respect to the noncompact actions, translations and dilatations implies that $\Lambda_{I}(\boldsymbol{M})=0$ is a necessary condition for boundedness below of $\Psi_{\mathbb{R}^{2}}$. Using similar techniques to those used in the proof of Theorem 2 , we obtain an analogous result concerning the boundedness of the functional. In addition, we establish an existence result for minimizers.

Theorem 4. Let $A$ be a symmetric matrix satisfying 2.1] and $M \in \mathbb{R}_{+}^{n}$. Then:

(i) $\Lambda_{I}(\boldsymbol{M})=0$ and 2.16 are necessary and sufficient conditions for the boundedness from below of $\Psi_{\mathbb{R}^{2}}$ on $\boldsymbol{\Gamma}_{M}\left(\mathbb{R}^{2}\right)$.

(ii) There exists a minimizer $\rho$ for $\Psi_{\mathbb{R}^{2}}$ over $\Gamma_{M}\left(\mathbb{R}^{2}\right)$ if and only if

$$
\Lambda_{I}(\boldsymbol{M})=0 \quad \text { and } \quad \Lambda_{J}(\boldsymbol{M})>0, \quad \forall J \varsubsetneqq I .
$$

Finally we turn to a version of the Moser-Trudinger inequality for systems on bounded domains. By Moser's inequality [13],

$$
\frac{1}{2} \int_{\Omega}|\nabla u|^{2}-8 \pi \log \left(\int_{\Omega} e^{u}\right) \geq-C, \quad \forall u \in H_{0}^{1}(\Omega),
$$

where $\Omega$ is a bounded domain in $\mathbb{R}^{2}$. The extension to systems is expected to take the form

$$
\frac{1}{2} \sum_{i, j \in I} \int_{\Omega} a_{i, j} \nabla u_{i} \nabla u_{j}-\sum_{i \in I} M_{i} \log \left(\int_{\Omega} \exp \left(\sum_{j \in I} a_{i, j} u_{j}\right)\right) \geq-C, \quad \forall \boldsymbol{u} \in\left(H_{0}^{1}(\Omega)\right)^{n},
$$

where $A$ is a matrix satisfying 2.1]. In [7] it was shown that 2.28) holds in the subcritical case (1.9). The question whether the same result remains valid in the critical case was left open. Our last theorem provides a positive answer to that question. Here again we use a dual formulation, involving the Green function $G_{\Omega}(x, y)$ for the operator $-\Delta$ on $\Omega$ with Dirichlet boundary conditions.

Theorem 5. Let $\Omega$ be a bounded domain in $\mathbb{R}^{2}$, A a symmetric matrix and $\boldsymbol{M} \in \mathbb{R}_{+}^{n}$. Then: 
(i) If A satisfies (2.1) then condition 2.16) is necessary and sufficient for the boundedness from below of

$$
\Psi_{\Omega}(\boldsymbol{\rho})=\sum_{i \in I} \int_{\Omega} \rho_{i}(x) \log \rho_{i}(x) d x-\frac{1}{2} \sum_{i, j \in I} a_{i, j} \int_{\Omega} \int_{\Omega} \rho_{i}(x) G_{\Omega}(x, y) \rho_{j}(y) d x d y
$$

over

$$
\boldsymbol{\Gamma}_{\boldsymbol{M}}(\Omega)=\left\{\boldsymbol{\rho}=\left(\rho_{i}\right)_{i \in I}: \rho_{i} \geq 0, \int_{\Omega} \rho_{i} \log \rho_{i}<\infty \text { and } \int_{\Omega} \rho_{i}=M_{i}, \forall i\right\} .
$$

If, in addition, the matrix $A$ is positive definite, then condition 2.16 is necessary and sufficient for (2.28) to hold.

(ii) More generally, if A corresponds to a collaborating system with $K$ blocks, then 2.21) is a necessary and sufficient condition for the boundedness from below of $\Psi_{\Omega}$ on $\Gamma_{M}(\Omega)$, and when $A$ is positive definite, for the validity of 2.28 .

\section{On duality and conformal invariance}

In this section we explore the two important notions of duality and conformal invariance. In particular, we shall prove Theorem 1 on the conformal case.

We begin by presenting a duality principle which connects the Moser-Trudinger functional $F^{M}$ with $\Psi_{S^{2}}$. An analogous statement for the problem on a bounded domain was proved in [7, Proposition 2.1]. A simple adaptation of the argument yields the result for our context too, but we prefer to present a slightly different approach which involves the functional

$$
\Phi(\boldsymbol{\rho}, \boldsymbol{u})=\sum_{i \in I} \int_{S^{2}} \rho_{i} \log \rho_{i}+\sum_{i, j \in I} a_{i, j}\left[\int_{S^{2}} \frac{1}{2} \nabla u_{i} \cdot \nabla u_{j}-\rho_{i} u_{j}\right]
$$

defined on $\boldsymbol{\Gamma}_{\boldsymbol{M}}\left(S^{2}\right) \times \mathcal{H}_{n}\left(S^{2}\right)$.

Lemma 3.1. Let $A$ be a symmetric $n \times n$ matrix. Then, for any fixed $\boldsymbol{u} \in \mathcal{H}_{n}\left(S^{2}\right), \Phi(\cdot, \boldsymbol{u})$ is bounded from below on $\boldsymbol{\Gamma}_{\boldsymbol{M}}\left(S^{2}\right)$ and

$$
\inf _{\boldsymbol{\rho} \in \boldsymbol{\Gamma}_{\boldsymbol{M}}\left(S^{2}\right)} \Phi(\boldsymbol{\rho}, \boldsymbol{u})=F^{\boldsymbol{M}}(\boldsymbol{u})+\sum_{i \in I} M_{i} \log \frac{M_{i}}{4 \pi} .
$$

Moreover, the infimum in 3.1) is uniquely attained at $\bar{\rho}$ given by

$$
\bar{\rho}_{i}=M_{i} \frac{\exp \left(\sum_{j \in I} a_{i, j} u_{j}\right)}{\int_{S^{2}} \exp \left(\sum_{j \in I} a_{i, j} u_{j}\right)}, \quad \forall i \in I \text {. }
$$


Proof. Applying the elementary inequality

$$
a b \leq b \log b-b+e^{a}, \quad \forall a \in \mathbb{R}, \forall b \in \mathbb{R}_{+},
$$

with $a=u_{j}(x) / \gamma$ and $b=\gamma \rho_{i}(x)$, and using (1.1), we find that for all $i, j \in I$ and $\gamma>0$,

$\int_{S^{2}} \rho_{i} u_{j} \leq \gamma \int_{S^{2}} \rho_{i} \log \rho_{i}+\int_{S^{2}} e^{u_{j} / \gamma}+\gamma M_{i} \log (\gamma / e) \leq \gamma \int_{S^{2}} \rho_{i} \log \rho_{i}+C\left(u_{j}, M_{i}, \gamma\right)$.

Therefore, for some $\varepsilon>0$ and $C=C(\boldsymbol{u}, \boldsymbol{M}, \varepsilon)$ we have

$$
\Phi(\boldsymbol{\rho}, \boldsymbol{u}) \geq \varepsilon \sum_{i \in I} \int_{S^{2}} \rho_{i} \log \rho_{i}-C .
$$

Hence, for each fixed $\boldsymbol{u} \in \mathcal{H}_{n}\left(S^{2}\right)$ the functional $\Phi(\cdot, \boldsymbol{u})$, which is continuous and strictly convex on the closed and convex subset $\boldsymbol{\Gamma}_{\boldsymbol{M}}\left(S^{2}\right)$ of the reflexive Banach space (Orlicz space)

$$
X=\left\{\boldsymbol{\rho}=\left(\rho_{i}\right)_{i \in I}: \int_{S^{2}}\left(\left(1+\left|\rho_{i}\right|\right) \log \left(1+\left|\rho_{i}\right|\right)-\left|\rho_{i}\right|\right)<\infty, \forall i\right\}
$$

satisfies the coercivity condition

$$
\Phi(\boldsymbol{\rho}, \boldsymbol{u}) \rightarrow \infty \quad \text { as } \sum_{i \in I} \int_{S^{2}} \rho_{i} \log \rho_{i} \rightarrow \infty, \quad \text { for } \boldsymbol{\rho} \in \boldsymbol{\Gamma}_{\boldsymbol{M}}\left(S^{2}\right)
$$

It follows that the minimum of $\Phi(\cdot, \boldsymbol{u})$ over $\boldsymbol{\Gamma}_{\boldsymbol{M}}\left(S^{2}\right)$ is attained, and the unique minimizer $\rho$ must satisfy (3.2). Plugging it in $\Phi$ we are led to 3.1 .

Next we examine the infimum of $\Phi(\boldsymbol{\rho}, \cdot)$ with respect to $\boldsymbol{u}$, for a fixed $\boldsymbol{\rho}$, under the additional assumption that $A$ is positive definite.

Lemma 3.2. Suppose A is positive definite. Then, for any fixed $\rho \in \Gamma_{\boldsymbol{M}}\left(S^{2}\right)$, the infimum of $\Phi(\boldsymbol{\rho}, \cdot)$ over $\mathcal{H}_{n}\left(S^{2}\right)$ is attained at $\overline{\boldsymbol{u}}$ given by

$$
-\Delta \bar{u}_{i}=\rho_{i}-\frac{M_{i}}{4 \pi} \quad \text { in } S^{2}, \forall i \in I .
$$

Moreover,

$$
\begin{aligned}
\min _{\boldsymbol{u} \in \mathcal{H}_{n}\left(S^{2}\right)} & \Phi(\boldsymbol{\rho}, \boldsymbol{u})=\sum_{i \in I} \int_{S^{2}} \rho_{i} \log \rho_{i} \\
& +\frac{1}{4 \pi} \sum_{i, j \in I} a_{i, j} \int_{S^{2}} \int_{S^{2}}\left(\rho_{i}(x)-\frac{M_{i}}{4 \pi}\right)(\log |x-y|)\left(\rho_{j}(y)-\frac{M_{j}}{4 \pi}\right) d x d y .
\end{aligned}
$$


Proof. Similarly to the proof of Lemma 3.1 we deduce that $\Phi(\rho, \cdot)$ is strictly convex and coercive over $\mathcal{H}_{n}\left(S^{2}\right)$ (here we use the assumption that $A$ is positive definite), and it follows that the minimum is attained at a unique $\boldsymbol{u} \in \mathcal{H}_{n}\left(S^{2}\right)$. Taking the variation of $\Phi(\boldsymbol{\rho}, \boldsymbol{u})$ with respect to each $u_{j}$ yields $\left[3.4\right.$. The Green function $G(x, y)$ for $-\Delta$ on $S^{2}$ is by definition a solution to

$$
-\Delta_{y} G(x, y)=\delta_{x}-\frac{1}{4 \pi} \quad \text { on } S^{2} .
$$

The solution to 3.6 is unique up to an additive constant, and we can choose as a representative $G(x, y)=-\frac{1}{2 \pi} \log |x-y|$ (here again $|x-y|$ stands for the Euclidean distance in $\mathbb{R}^{3}$ ). Therefore, we may rewrite 3.4 as

$$
\bar{u}_{i}=G *\left(\rho_{i}-\frac{M_{i}}{4 \pi}\right) \quad \forall i \in I .
$$

Plugging 3.7 in $\Phi$ and using the equality

$$
\begin{aligned}
\int_{S^{2}}\left(\frac{1}{2} \nabla \bar{u}_{i} \cdot \nabla \bar{u}_{j}-\rho_{i} \bar{u}_{j}\right) & =\int_{S^{2}}\left(\frac{1}{2}\left(\rho_{i}-\frac{M_{i}}{4 \pi}\right) \bar{u}_{j}-\rho_{i} \bar{u}_{j}\right) \\
& =-\frac{1}{2} \int_{S^{2}} \int_{S^{2}}\left(\rho_{i}(x)-\frac{M_{i}}{4 \pi}\right) G(x, y)\left(\rho_{j}(y)-\frac{M_{j}}{4 \pi}\right) d x d y,
\end{aligned}
$$

we are led to 3.5 .

A simple corollary of the above is the following analogue to [7, Proposition 2.1].

Corollary 3.1. Let $A=\left(a_{i, j}\right)$ be a positive definite matrix. Then $\Psi_{S^{2}}$ is bounded from below on $\boldsymbol{\Gamma}_{\boldsymbol{M}}\left(S^{2}\right)$ iff $F^{M}$ is bounded from below on $\mathcal{H}_{n}\left(S^{2}\right)$ and

$$
\inf _{\boldsymbol{\rho} \in \boldsymbol{\Gamma}_{\boldsymbol{M}}\left(S^{2}\right)} \Psi_{S^{2}}(\boldsymbol{\rho})=\inf _{\boldsymbol{u} \in \mathcal{H}_{n}\left(S^{2}\right)} F^{\boldsymbol{M}}(\boldsymbol{u})+\sum_{i \in I} M_{i} \log \frac{M_{i}}{4 \pi}+\frac{c_{0}}{16 \pi^{2}} \sum_{i, j \in I} a_{i, j} M_{i} M_{j}
$$

where $c_{0}$ is defined in 2.24). Moreover, existence of minimizers for the two problems is equivalent, and the minimizers are related via (3.2) and (3.4).

Proof. The result follows immediately from Lemmas 3.1 and 3.2 by using

$$
\inf _{\boldsymbol{u} \in \mathcal{H}_{n}\left(S^{2}\right)} \inf _{\boldsymbol{\rho} \in \boldsymbol{\Gamma}_{\boldsymbol{M}}\left(S^{2}\right)} \Phi(\boldsymbol{\rho}, \boldsymbol{u})=\inf _{\boldsymbol{\rho} \in \boldsymbol{\Gamma}_{\boldsymbol{M}}\left(S^{2}\right)} \inf _{\boldsymbol{u} \in \mathcal{H}_{n}\left(S^{2}\right)} \Phi(\boldsymbol{\rho}, \boldsymbol{u}) .
$$

Next we turn to the notion of conformal invariance and present the simple proof of Theorem 1 concerning the conformal and subconformal cases.

Proof of Theorem 1. By the duality principle of Corollary 3.1 it follows that, for positive definite $A, 2.13$ is equivalent to 2.15). Indeed, it suffices to note that the function $w=$ $\log \left|\mathcal{J}_{\tau}\right|$ satisfies the equation

$$
-\Delta w=2\left(e^{w}-1\right)=2\left(\left|\mathcal{J}_{\tau}\right|-1\right) .
$$


Hence, for $\rho_{i}=\frac{M_{i}}{4 \pi}\left|\mathcal{J}_{\tau}\right|$, 3.4 gives $\bar{u}_{i}=\frac{M_{i}}{8 \pi} \log \left|\mathcal{J}_{\tau}\right|+c_{i}$. As explained in Section 2 , 2.13) is equivalent to 2.14 in the conformal case. It suffices thus to prove the assertions about $\Psi_{S^{2}}$. We recall the following form of the logarithmic Hardy-Littlewood-Sobolev inequality (see [3, Theorem 2]; it is equivalent to the critical case of (1.3):

$$
\begin{aligned}
\int_{S^{2}} F \log F+\int_{S^{2}} G \log G+\frac{1}{\pi} \int_{S^{2}} \int_{S^{2}} F(x)(\log |x-y|) G(y) d x d y \\
\geq 4 c_{0}, \quad \forall F, G \in \Gamma_{4 \pi}\left(S^{2}\right)
\end{aligned}
$$

with equality iff $F$ and $G$ both equal the same conformal image of the constant function $H \equiv 1$. Applying (3.9) to $F=\frac{4 \pi}{M_{i}} \rho_{i}$ and $G=\frac{4 \pi}{M_{j}} \rho_{j}$ yields, for each $i, j \in I$,

$$
\begin{gathered}
M_{j} \int_{S^{2}} \rho_{i} \log \rho_{i}+M_{i} \int_{S^{2}} \rho_{j} \log \rho_{j}+4 \int_{S^{2}} \int_{S^{2}} \rho_{i}(x)(\log |x-y|) \rho_{j}(y) d x d y \\
\geq \frac{M_{i} M_{j}}{\pi} c_{0}+M_{i} M_{j} \log \frac{M_{i} M_{j}}{16 \pi^{2}} .
\end{gathered}
$$

Multiplying (3.10) by $\frac{a_{i, j}}{16 \pi}$ and summing on $i, j$ yields

$$
\begin{gathered}
\sum_{i \in I} \frac{\sum_{j \in I} a_{i, j} M_{j}}{8 \pi} \int_{S^{2}} \rho_{i} \log \rho_{i}+\frac{1}{4 \pi} \sum_{i, j \in I} a_{i, j} \int_{S^{2}} \int_{S^{2}} \rho_{i}(x)(\log |x-y|) \rho_{j}(y) d x d y \\
\geq \frac{c_{0}}{16 \pi^{2}} \sum_{i, j \in I} a_{i, j} M_{i} M_{j}+\frac{1}{16 \pi} \sum_{i, j \in I} a_{i, j} M_{i} M_{j} \log \frac{M_{i} M_{j}}{16 \pi^{2}} \\
=\frac{c_{0}}{16 \pi^{2}} \sum_{i, j \in I} a_{i, j} M_{i} M_{j}+\sum_{i \in I}\left(1-\frac{\nu_{i}}{2}\right) M_{i} \log \frac{M_{i}}{4 \pi}
\end{gathered}
$$

which may be rewritten as

$$
\Psi_{S^{2}}(\rho) \geq \Psi_{S^{2}}\left(\rho^{0}\right)+\sum_{i \in I} \frac{v_{i}}{2} \int_{S^{2}} \rho_{i} \log \left(4 \pi \rho_{i} / M_{i}\right),
$$

where $v_{i}$ is defined in 2.7). In the conformal case, $v_{i}=0$ for all $i \in I$, and 2.13 follows from (3.12). Moreover, (ii) follows from conformal invariance, and the uniqueness assertion for this case in (v) follows from the characterization of uniqueness in (3.9).

In the subconformal case $\left(v_{i} \geq 0\right.$ for all $i$ and $v_{i_{0}}>0$ for some $\left.i_{0}\right)$ we apply Jensen's inequality

$$
\int_{S^{2}}\left(4 \pi \rho_{i} / M_{i}\right) \log \left(4 \pi \rho_{i} / M_{i}\right) \geq 0
$$

to the r.h.s. of 3.12 to infer that

$$
\Psi_{S^{2}}(\rho) \geq \Psi_{S^{2}}\left(\rho^{0}\right) .
$$

For $\rho^{0}$ there is equality in 3.14. Therefore, $\rho^{0}$ is a minimizer. Moreover, if $\rho$ is any minimizer, then equality must hold in 3.10 for all $i, j \in I$ such that $a_{i, j}>0$. Under the 
assumption that $A$ is irreducible, it follows that $\rho$ is a conformal image of $\rho^{0}$. We claim that actually $\rho=\rho^{0}$. Indeed, assume by negation that $\rho$ is a nontrivial conformal image of $\rho^{0}$, and thus all its components are nonconstant. This yields strict inequality in 3.13 for all $i \in I$. By assumption there is an $i_{0} \in I$ for which $v_{i_{0}}>0$, and therefore, by 3.12 the inequality in 3.14 too is strict. Contradiction.

Our next result shows that the minimizing property of the constant configuration $\rho^{0}$ characterizes the conformal case among the critical cases. More precisely, we have

Proposition 3.1. Let A be a symmetric matrix satisfying 2.1. Assume that $\Lambda_{I}(\boldsymbol{M})=0$ but that (2.11) is not satisfied, i.e., there exists $i_{0}$ such that $v_{i_{0}} \neq 0$. Then $\rho^{0}$ is not $a$ minimizer for $\Psi_{S^{2}}$ over $\boldsymbol{\Gamma}_{\boldsymbol{M}}\left(S^{2}\right)$.

Proof. Assume by negation that $\boldsymbol{\rho}^{0}$ is a minimizer. Then also $\tilde{\boldsymbol{\rho}}^{0}$ is a minimizer for $\widetilde{\Psi}_{\mathbb{R}^{2}}$ over $\boldsymbol{\Gamma}_{\boldsymbol{M}}\left(\mathbb{R}^{2}\right)$. Using our assumption $\Lambda_{I}(\boldsymbol{M})=0$ (i.e. $\sum_{i \in I} v_{i} M_{i}=0$ ) and the fact that the components of $\tilde{\boldsymbol{\rho}}^{0}$ are proportional to each other in $(2.10)$ yields

$$
\Psi_{\mathbb{R}^{2}}\left(\tilde{\boldsymbol{\rho}}^{0}\right)=\widetilde{\Psi}_{\mathbb{R}^{2}}\left(\tilde{\boldsymbol{\rho}}^{0}\right) .
$$

Moreover, whenever $\Lambda_{I}(\boldsymbol{M})=0$ we also have

$$
\inf _{\Gamma_{M}\left(\mathbb{R}^{2}\right)} \widetilde{\Psi}_{\mathbb{R}^{2}} \leq \inf _{\Gamma_{\boldsymbol{M}}\left(\mathbb{R}^{2}\right)} \Psi_{\mathbb{R}^{2}}
$$

Indeed, fix any $\tilde{\boldsymbol{\rho}} \in \boldsymbol{\Gamma}_{\boldsymbol{M}}\left(\mathbb{R}^{2}\right)$. Then the functional $\Psi_{\mathbb{R}^{2}}$ is invariant with respect to dilatations, i.e. $\Psi_{\mathbb{R}^{2}}\left(\tilde{\boldsymbol{\rho}}^{(\alpha)}\right)=\Psi_{\mathbb{R}^{2}}(\tilde{\boldsymbol{\rho}})$ for all $\alpha>0$, where $\tilde{\boldsymbol{\rho}}^{(\alpha)}(x)=\alpha^{2} \tilde{\boldsymbol{\rho}}(\alpha x)$. Moreover,

$$
\lim _{\alpha \rightarrow \infty} \int_{\mathbb{R}^{2}} \tilde{\rho}_{i}^{(\alpha)} \log \left(1+|x|^{2}\right) d x=\lim _{\alpha \rightarrow \infty} \int_{\mathbb{R}^{2}} \tilde{\rho}_{i} \log \left(1+|x / \alpha|^{2}\right) d x=0, \quad \forall i \in I,
$$

and thus $\lim _{\alpha \rightarrow \infty} \widetilde{\Psi}_{\mathbb{R}^{2}}\left(\tilde{\boldsymbol{\rho}}^{(\alpha)}\right)=\Psi_{\mathbb{R}^{2}}(\tilde{\boldsymbol{\rho}})$. This clearly implies 3.16). Combining 3.16 with 3.15 it follows that $\tilde{\rho}^{0}$ is also a minimizer of $\Psi_{\mathbb{R}^{2}}$ over $\Gamma_{M}\left(\mathbb{R}^{2}\right)$. However, every minimizer $\tilde{\boldsymbol{\rho}}$ of $\widetilde{\Psi}_{\mathbb{R}^{2}}$ over $\Gamma_{\boldsymbol{M}}\left(\mathbb{R}^{2}\right)$ satisfies the Euler-Lagrange equations

$$
\tilde{\rho}_{i}(x)=\lambda_{i}\left(1+|x|^{2}\right)^{-v_{i}} \exp \left(-\frac{1}{2 \pi} \sum_{j \in I} a_{i, j} \int_{\mathbb{R}^{2}} \rho_{j}(y) \log |x-y| d y\right), \quad \forall i \in I,
$$

for some positive constants $\lambda_{1}, \ldots, \lambda_{n}$. On the other hand, any minimizer of $\Psi_{\mathbb{R}^{2}}$ satisfies 3.17) with $v_{i}=0$ for all $i \in I$. Since $\tilde{\boldsymbol{\rho}}^{0}$ is a minimizer for both functionals, we get a contradiction for $i=i_{0}$.

\section{A basic estimate}

Proposition 4.1 below provides the main tool for the proofs of Theorems 2,4 and 5 . Since in all these results condition (2.16) plays an important role, we begin with an interpretation of it. The proof of the following elementary lemma requires a simple modification of the proof of [7, Lemma 5.1]. 
Lemma 4.1. Let $A$ be a symmetric matrix and let $\boldsymbol{M} \in \mathbb{R}_{+}^{n}$ satisfy $\Lambda_{I}(\boldsymbol{M})=0$. Then for each $i \in I$ the following two conditions are equivalent:

$$
\begin{gathered}
\frac{\partial \Lambda_{I}}{\partial M_{i}}(\boldsymbol{M})<0, \\
\Lambda_{I \backslash\{i\}}(\boldsymbol{M})+a_{i, i} M_{i}^{2}>0 .
\end{gathered}
$$

Proof. Since $\frac{\partial \Lambda_{I}}{\partial M_{i}}(\boldsymbol{M})=8 \pi-2 \sum_{j \in I} a_{i, j} M_{j}$, we have

$$
\begin{aligned}
0=\Lambda_{I}(\boldsymbol{M}) & =\Lambda_{I \backslash\{i\}}(\boldsymbol{M})+a_{i, i} M_{i}^{2}+M_{i}\left(8 \pi-2 \sum_{j \in I} a_{i, j} M_{j}\right) \\
& =\Lambda_{I \backslash\{i\}}(\boldsymbol{M})+a_{i, i} M_{i}^{2}+M_{i} \frac{\partial \Lambda_{I}}{\partial M_{i}}(\boldsymbol{M}),
\end{aligned}
$$

and the result follows.

The next lemma explains the significance of condition 2.16.

Lemma 4.2. Let $A$ be a symmetric matrix with $a_{i, i} \geq 0$ for all $i \in I$, and let $\boldsymbol{M} \in \mathbb{R}_{+}^{n}$ satisfy (1.10). Then 2.16) is satisfied if and only if $\Lambda_{I}$ is identically zero on no edge of the box

$$
\mathcal{B}(\boldsymbol{M})=\left\{\boldsymbol{N} \in \mathbb{R}_{+}^{n}: \boldsymbol{N} \leq \boldsymbol{M}\right\} .
$$

Proof. The $2^{n}$ vertices of $\mathcal{B}(\boldsymbol{M})$ are $\mathbf{0}$ and $\left\{\boldsymbol{M}_{J}\right\}_{\emptyset \neq J \subseteq I}$ where

$$
\left(\boldsymbol{M}_{J}\right)_{j}= \begin{cases}M_{j} & \text { if } j \in J, \\ 0 & \text { otherwise. }\end{cases}
$$

Assume first that 2.16) is not satisfied. Then there exist $J \subseteq I$ and $i \in J$ such that $\Lambda_{J}(\boldsymbol{M})=0, a_{i, i}=0$ and $\Lambda_{K}(\boldsymbol{M})=0$ for $K=J \backslash\{i\}$. Since $a_{j, j} \geq 0$ for all $j \in I$, and $\Lambda_{I} \geq 0$ on all the vertices of $\mathcal{B}(\boldsymbol{M})$ (by 1.10 ), it follows from the maximum principle that $\Lambda_{I} \geq 0$ on $\mathcal{B}(\boldsymbol{M})$. Let $f_{J}:\left[0, M_{i}\right] \rightarrow[0, \infty)$ denote the restriction of $\Lambda_{I}$ to the edge connecting $\boldsymbol{M}_{K}$ to $\boldsymbol{M}_{J}$. Since $a_{i, i}=0, f_{J}$ is a linear function on $\left[0, M_{i}\right]$ satisfying $f_{J}\left(M_{i}\right)=\Lambda_{J}(\boldsymbol{M})=0$ and $f_{J}^{\prime}\left(M_{i}\right)=0$ (by Lemma 4.1). Therefore $f_{J} \equiv 0$ on $\left[0, M_{i}\right]$, i.e. $\Lambda_{I}=0$ on the edge connecting $\boldsymbol{M}_{K}$ to $\boldsymbol{M}_{J}$.

Assume next that $\Lambda_{I}$ is identically zero on some edge connecting $\boldsymbol{M}_{K}$ to $\boldsymbol{M}_{J}$ with $J=K \cup\{i\}$. Clearly $\Lambda_{I}\left(\boldsymbol{M}_{J}\right)=\Lambda_{I}\left(\boldsymbol{M}_{K}\right)=0$ and $\frac{\partial \Lambda_{I}}{\partial M_{i}}\left(\boldsymbol{M}_{J}\right)=0$. Therefore, by Lemma 4.1 we infer that $\Lambda_{K}(\boldsymbol{M})=a_{i, i}=0$ and 2.16) fails to hold.

Now we are in a position to present the main result of this section.

Proposition 4.1. Let $A$ be a symmetric matrix with $a_{i, i} \geq 0$ for all $i \in I$, and let $\boldsymbol{M} \in \mathbb{R}_{+}^{n}$. Then there exists a constant $C_{0}$ such that

$$
\int_{-\infty}^{0} \sum_{i \in I} w_{i}^{\prime} \log w_{i}^{\prime} d s+\frac{1}{4 \pi} \int_{-\infty}^{0} \Lambda_{I}(\boldsymbol{w}) d s \geq-C_{0}
$$


for all $\boldsymbol{w}=\left(w_{1}, \ldots, w_{n}\right)$ whose components are absolutely continuous on $\mathbb{R}_{-}$and satisfy

$$
w_{i}^{\prime} \geq 0 \quad \text { on } \quad(-\infty, 0), \quad \lim _{s \rightarrow-\infty} w_{i}(s)=0 \quad \text { and } \quad w_{i}(0)=M_{i}, \quad \forall i,
$$

if and only if $\boldsymbol{M}$ satisfies condition 2.16 .

Proof. (i) We first prove the sufficiency of condition 2.16. Put

$$
F\left(\boldsymbol{w}, \boldsymbol{w}^{\prime}\right)=\sum_{i \in I} w_{i}^{\prime} \log w_{i}^{\prime}+\frac{1}{4 \pi} \Lambda_{I}(\boldsymbol{w}) .
$$

Applying the elementary inequality (3.3) with $a=\log \frac{\Lambda_{I}(w)}{4 \pi n}$ and $b=w_{i}^{\prime}$ yields for each $s \in(-\infty, 0)$,

$$
w_{i}^{\prime} \log w_{i}^{\prime}+\frac{\Lambda_{I}(\boldsymbol{w})}{4 \pi n} \geq w_{i}^{\prime}\left[\log \Lambda_{I}(\boldsymbol{w})-\log 4 \pi n+1\right] .
$$

Therefore,

$$
\int_{-\infty}^{0} F\left(\boldsymbol{w}, \boldsymbol{w}^{\prime}\right) d s \geq \int_{-\infty}^{0}\left(\sum_{i \in I} w_{i}^{\prime}\right) \log \Lambda_{I}(\boldsymbol{w}) d s-\log \left(\frac{4 \pi n}{e}\right) \sum_{i \in I} M_{i} .
$$

For each $i$ we have

$$
\int_{-\infty}^{0} w_{i}^{\prime} \log \Lambda_{I}(\boldsymbol{w}) d s \geq \int_{-\infty}^{0} w_{i}^{\prime} \log \lambda_{i}\left(w_{i}\right) d s=\int_{0}^{M_{i}} \log \lambda_{i}(m) d m
$$

where

$$
\lambda_{i}(m):=\inf \left\{\Lambda_{I}(\boldsymbol{N}): \boldsymbol{N}=\left(N_{1}, \ldots, N_{n}\right) \in \mathcal{B}(\boldsymbol{M}), N_{i}=m\right\} .
$$

Since $a_{j, j} \geq 0$ for all $j$, the minimum in (4.7) is attained at one of the vertices of the $n$-1-dimensional box $\left\{\boldsymbol{N} \in \mathcal{B}(\boldsymbol{M}): N_{i}=m\right\}$.

Let us fix any $i \in I$. Setting for each $J \varsubsetneqq I(J=\emptyset$ is allowed, and we denote $\left.\boldsymbol{M}_{\emptyset}=\mathbf{0}\right)$ and $j \notin J$,

$$
g_{J, j}(m)=\Lambda_{I}\left(\left(1-m / M_{j}\right) \boldsymbol{M}_{J}+\left(m / M_{j}\right) \boldsymbol{M}_{J \cup\{j\}}\right) \quad \text { for } m \in\left[0, M_{j}\right],
$$

we then have

$$
\lambda_{i}(m)=\min \left\{g_{J, i}(m): J \subseteq I \backslash\{i\}\right\} \quad \text { for } m \in\left[0, M_{i}\right] .
$$

For each $J \subseteq I \backslash\{i\}, g_{J, i}$ is a concave quadratic polynomial in the variable $m$ (since $\left.a_{i, i} \geq 0\right)$ which is nonnegative on $\left[0, M_{i}\right]$. If $g_{J, i}(0)=0$ then we must have $g_{J, i}^{\prime}(0)>0$. Indeed, $g_{J, i}^{\prime}(0)=0$ would imply that $\Lambda_{I}$ is identically zero on the edge joining $M_{J}$ to $M_{J \cup\{i\}}$, contradicting 2.16 and Lemma 4.2 By the same argument we have: either $g_{J, i}\left(M_{i}\right)>0$, or, if $g_{J, i}\left(M_{i}\right)=0$, then $g_{J, i}\left(M_{i}\right)<0$. We conclude that there exists $\alpha_{J, i}>0$ such that

$$
g_{J, i}(m) \geq \alpha_{J, i} m\left(M_{i}-m\right) \quad \text { on }\left[0, M_{i}\right] .
$$


In view of 4.8 , we obtain for $\bar{\alpha}_{i}:=\min \left\{\alpha_{J, i}: J \subseteq I \backslash\{i\}\right\}$,

$$
\lambda_{i}(m) \geq \bar{\alpha}_{i} m\left(M_{i}-m\right) \quad \text { on }\left[0, M_{i}\right] .
$$

Clearly,

$$
\int_{0}^{M_{i}} \log \left(m\left(M_{i}-m\right)\right) d m>-\infty,
$$

and (4.3) follows from 4.5), (4.6), 4.9) and (4.10).

(ii) Next we prove the necessity of (2.16). Assume that 2.16 is not satisfied. If $\Lambda_{J}(\boldsymbol{M})<0$ for some $\emptyset \neq J \subseteq I$, then by the argument of [7, Lemma 2.2] it follows that (4.3) cannot hold. Assume then that (1.10) is satisfied, but for some $i \in I$ and $\emptyset \neq K \subseteq I \backslash\{i\}$ we have, for $J=K \cup\{i\}$,

$$
\Lambda_{I}\left(\boldsymbol{M}_{K}\right)=\Lambda_{I}\left(\boldsymbol{M}_{J}\right)=a_{i, i}=0 .
$$

Note that $\Lambda_{I}\left(\boldsymbol{M}_{J}\right)=a_{i, i}=0$ implies that $J$ is not a singleton, i.e. $K \neq \emptyset$. Then by Lemma 4.2 it follows that $\Lambda_{I}$ is identically zero on the edge connecting $\boldsymbol{M}_{K}$ to $\boldsymbol{M}_{J}$. For each $m$ we construct a path $z_{m}:(-\infty, 0) \rightarrow \mathcal{B}(\boldsymbol{M})$ connecting $\mathbf{0}$ to $\boldsymbol{M}$ as follows. For simplicity we omit the subscript $m$. First, on $(-\infty,-m-1)$ we set

$$
z_{j}(t)= \begin{cases}M_{j} e^{t+m+1} & \text { if } j \in K, \\ 0 & \text { if } j \notin K .\end{cases}
$$

Then

$$
\int_{-\infty}^{-m-1} F\left(z, z^{\prime}\right) d t=c_{1}
$$

for some constant $c_{1}$ independent of $m$. On $[-m-1,-1]$ we connect $\boldsymbol{M}_{K}$ to $\boldsymbol{M}_{J}$ by setting

$$
z_{j}(t)= \begin{cases}M_{j} & \text { if } j \in K, \\ M_{i} t / m & \text { if } j=i, \\ 0 & \text { if } j \notin J\end{cases}
$$

Then

$$
\int_{-m-1}^{-1} F\left(z, z^{\prime}\right) d t=\int_{-m-1}^{-1} z_{i}^{\prime} \log z_{i}^{\prime} d t=M_{i} \log \frac{M_{i}}{m} \rightarrow-\infty \quad \text { as } m \rightarrow \infty .
$$

Finally, on $[-1,0]$ we connect $\boldsymbol{M}_{J}$ to $\boldsymbol{M}$ by $\boldsymbol{z}(t)=(-t) \boldsymbol{M}_{J}+(1+t) \boldsymbol{M}$, which gives

$$
\int_{-1}^{0} F\left(z, z^{\prime}\right) d t=c_{2}
$$

Combining (4.12)-(4.14) yields

$$
\lim _{m \rightarrow \infty} \int_{-\infty}^{0} F\left(z_{m}, z_{m}^{\prime}\right) d t=-\infty
$$

and thus (4.3) does not hold. 


\section{Proofs of the main results}

In this section we prove our main results, Theorems 245. We divide the assumptions on $A$ into two cases. The first case, of $A$ nonnegative (i.e. satisfying (2.1)), will be treated in Subsection 5.1. The second case, studied in Subsection 5.2, is of a multi-block collaborating system, i.e. when, up to a permutation of the indices of $I, A$ consists of $K \geq 2$ nonnegative blocks on the diagonal and outside these blocks all the elements are nonpositive (see (2.17)). The case of nonnegative $A$, corresponding to the case $K=1$, is then entitled: single-block collaborating system.

\subsection{Single-block collaborating systems}

We begin with the proof of Theorem 5(i), which is concerned with the problem on a bounded domain $\Omega \subset \mathbb{R}^{2}$. It extends [7, Lemma 2.1] to the critical case.

Proof of Theorem 5 $i$ ). By the duality principle of [7, Proposition 2.1] proving boundedness of $\Psi_{\Omega}$ will imply (2.28). Using Schwarz symmetrization as in the proof of [7] Lemma 2.1] we see that it suffices to consider the case where $\Omega=B_{R}$, the disc centered at 0 with radius $R$, and where each $\rho_{i}$ is radially symmetric and nonincreasing. We next sketch the argument from [7, Lemma 2.1] in order to obtain an equivalent expression for $\Psi_{\Omega}(\rho)$. Denoting, for each $i$, by $u_{i}$ the solution to $-\Delta u_{i}=\rho_{i}$ in $B_{R}$ with zero boundary condition we have

$$
\Psi_{\Omega}(\boldsymbol{\rho})=\sum_{i \in I} \int_{B_{R}} \rho_{i} \log \rho_{i} d x-\frac{1}{2} \sum_{i, j \in I} a_{i, j} \int_{B_{R}} \rho_{i} u_{j} d x
$$

Put $m_{i}(r)=\int_{B_{r}} \rho_{i} d x=2 \pi \int_{0}^{r} \tau \rho_{i}(\tau) d \tau$ so that $u_{i}^{\prime}(r)=-\frac{m_{i}(r)}{2 \pi r}$. Then

$$
\begin{aligned}
\int_{B_{R}} \rho_{i} \log \rho_{i} d x & =2 \pi \int_{0}^{R} \rho_{i} \log \rho_{i} r d r \\
& =\int_{0}^{R} m_{i}^{\prime} \log m_{i}^{\prime} d r-\int_{0}^{R} m_{i}^{\prime} \log r d r-m_{i}(R) \log (2 \pi) \\
& =\int_{0}^{R} m_{i}^{\prime} \log m_{i}^{\prime} d r+\int_{0}^{R} \frac{m_{i}}{r} d r-m_{i}(R) \log (2 \pi R)
\end{aligned}
$$

and

$$
\begin{aligned}
\int_{B_{R}} \rho_{i} u_{j} d x & =\int_{0}^{R} m_{i}^{\prime} u_{j} d r \\
& =\frac{1}{2 \pi} \int_{0}^{R} \frac{m_{i} m_{j}}{r} d r+m_{i}(R) u_{j}(R)=\frac{1}{2 \pi} \int_{0}^{R} \frac{m_{i} m_{j}}{r} d r .
\end{aligned}
$$


Plugging 5.2 -5.3 in 5.1) yields

$$
\begin{aligned}
\Psi_{\Omega}(\boldsymbol{\rho})= & \int_{0}^{R} \sum_{i \in I} m_{i}^{\prime} \log m_{i}^{\prime} d r \\
& +\int_{0}^{R}\left[\sum_{i \in I} \frac{m_{i}}{r}-\frac{1}{4 \pi} \sum_{i, j \in I} a_{i, j} \frac{m_{i} m_{j}}{r}\right] d r-\log (2 \pi R) \sum_{i \in I} M_{i} .
\end{aligned}
$$

Finally, setting $w_{i}(s)=m_{i}\left(e^{s}\right)$ for all $s \in(-\infty, \log R]$ and $i \in I$, we may rewrite [5.4) as

$$
\begin{aligned}
\Psi_{\Omega}(\boldsymbol{\rho})= & \int_{-\infty}^{\log R} \sum_{i \in I} w_{i}^{\prime} \log w_{i}^{\prime} d s \\
& +\int_{-\infty}^{\log R}\left[2 \sum_{i \in I} w_{i}-\frac{1}{4 \pi} \sum_{i, j \in I} a_{i, j} w_{i} w_{j}\right] d s-\log (2 \pi R) \sum_{i \in I} M_{i},
\end{aligned}
$$

and the result then follows directly from Proposition 4.1

Next we present the proof of the entropy inequality of Theorem 4

Proof of Theorem $4(i)$. We begin by proving sufficiency of the conditions. First we show that it is enough to consider radially symmetric $\tilde{\boldsymbol{\rho}}$. In fact, setting $\tilde{\boldsymbol{\rho}}^{*}=\left(\tilde{\rho}_{i}^{*}\right)_{i=1}^{n}$, where for each $i, \tilde{\rho}_{i}^{*}$ is the symmetric decreasing rearrangement of $\tilde{\rho}_{i}$, we clearly have

$$
\int_{\mathbb{R}^{2}} \tilde{\rho}_{i}^{*} \log \tilde{\rho}_{i}^{*} d x=\int_{\mathbb{R}^{2}} \tilde{\rho}_{i} \log \tilde{\rho}_{i} d x, \quad \int_{\mathbb{R}^{2}} \tilde{\rho}_{i}^{*}\left|\log \tilde{\rho}_{i}^{*}\right| d x=\int_{\mathbb{R}^{2}} \tilde{\rho}_{i}\left|\log \tilde{\rho}_{i}\right| d x,
$$

and

$$
\int_{\mathbb{R}^{2}} \tilde{\rho}_{i}^{*} \log \left(1+|x|^{2}\right) d x \leq \int_{\mathbb{R}^{2}} \tilde{\rho}_{i} \log \left(1+|x|^{2}\right) d x .
$$

In particular we deduce that $\tilde{\boldsymbol{\rho}}^{*} \in \boldsymbol{\Gamma}_{\boldsymbol{M}}\left(\mathbb{R}^{2}\right)$ whenever $\tilde{\boldsymbol{\rho}} \in \boldsymbol{\Gamma}_{\boldsymbol{M}}\left(\mathbb{R}^{2}\right)$. By a variant of the Riesz rearrangement inequality (see [4, Lemma 2]) we have

$\int_{\mathbb{R}^{2}} \int_{\mathbb{R}^{2}} \tilde{\rho}_{i}(x)(\log |x-y|) \tilde{\rho}_{j}(y) d x d y \geq \int_{\mathbb{R}^{2}} \int_{\mathbb{R}^{2}} \tilde{\rho}_{i}^{*}(x)(\log |x-y|) \tilde{\rho}_{j}^{*}(y) d x d y, \quad \forall i, j$.

Thus $\Psi_{\mathbb{R}^{2}}\left(\tilde{\boldsymbol{\rho}}^{*}\right) \leq \Psi_{\mathbb{R}^{2}}(\tilde{\boldsymbol{\rho}})$, and we may assume in what follows that each $\tilde{\rho}_{i}$ is a radially symmetric and decreasing function of $r=|x|$. For each $i$ let $-u_{i}$ denote the Newtonian potential of $\tilde{\rho}_{i}$, i.e.

$$
u_{i}(x)=-\frac{1}{2 \pi} \int_{\mathbb{R}^{2}} \tilde{\rho}_{i}(y) \log |x-y| d y .
$$

Thus $u_{i}(x)=u_{i}(r)$ is a radial function satisfying $-\Delta u_{i}=\tilde{\rho}_{i}$ in $\mathbb{R}^{2}$. Our assumption that $\int_{\mathbb{R}^{2}} \tilde{\rho}_{i}(x) \log \left(1+|x|^{2}\right) d x<\infty$ (cf. (2.8) implies that $\tilde{\rho}_{i}$ is regular at infinity (see [8, Ch. II, §3]), and in particular, since $\tilde{\rho}_{i}$ is radial, we have

$$
-u_{i}(r)=\frac{\log r}{2 \pi} \int_{B_{r}} \tilde{\rho}_{i}(y) d y+\frac{1}{2 \pi} \int_{\mathbb{R}^{2} \backslash B_{r}} \tilde{\rho}_{i}(y) \log |y| d y
$$


(see [8, Ch. II, §3, Lemma 9]). By (5.5) we deduce easily that

$$
\lim _{R \rightarrow \infty} u_{i}(R)+\frac{M_{i}}{2 \pi} \log R=0 .
$$

We define $m_{i}(r)=2 \pi r \int_{0}^{r} \tilde{\rho}_{i}(\tau) d \tau$ as above. Since for $R>1$,

$$
0 \leq(\log R)\left(M_{i}-m_{i}(R)\right)=(\log R) \int_{\mathbb{R}^{2} \backslash B_{R}} \tilde{\rho}_{i}(y) d y \leq \int_{\mathbb{R}^{2} \backslash B_{R}} \tilde{\rho}_{i}(y) \log |y| d y,
$$

we obtain

$$
\lim _{R \rightarrow \infty}\left(M_{i}-m_{i}(R)\right) \log R=0 .
$$

Clearly,

$$
\Psi_{\mathbb{R}^{2}}(\tilde{\boldsymbol{\rho}})=\lim _{R \rightarrow \infty} \bar{\Psi}_{R}(\tilde{\boldsymbol{\rho}})
$$

where

$$
\bar{\Psi}_{R}(\tilde{\boldsymbol{\rho}})=\sum_{i \in I} \int_{B_{R}} \tilde{\rho}_{i} \log \tilde{\rho}_{i} d x-\frac{1}{2} \sum_{i, j \in I} a_{i, j} \int_{B_{R}} \tilde{\rho}_{i} u_{j} d x
$$

Using (5.2) and the first two equalities in 5.3 yields $\bar{\Psi}_{R}(\tilde{\boldsymbol{\rho}})=G_{R}(\boldsymbol{m})-\log (2 \pi)$ $\cdot \sum_{i \in I} m_{i}(R)$ with

$$
\begin{aligned}
G_{R}(\boldsymbol{m})= & \int_{0}^{R} \sum_{i \in I} m_{i}^{\prime} \log m_{i}^{\prime} d r+\int_{0}^{R}\left[\sum_{i \in I} \frac{m_{i}}{r}-\frac{1}{4 \pi} \sum_{i, j \in I} a_{i, j} \frac{m_{i} m_{j}}{r}\right] d r \\
& -\sum_{i \in I} m_{i}(R)\left(\log R+\frac{1}{2} \sum_{j \in I} a_{i, j} u_{j}(R)\right) .
\end{aligned}
$$

Next, setting $w_{i}(s)=m_{i}\left(e^{s}\right)$ as above we get

$$
\begin{aligned}
G_{R}(\boldsymbol{m})= & \int_{-\infty}^{\log R} \sum_{i \in I} w_{i}^{\prime} \log w_{i}^{\prime} d s+\int_{-\infty}^{\log R}\left[2 \sum_{i \in I} w_{i}-\frac{1}{4 \pi} \sum_{i, j \in I} a_{i, j} w_{i} w_{j}\right] d s \\
& -\sum_{i \in I} m_{i}(R)\left(2 \log R+\frac{1}{2} \sum_{j \in I} a_{i, j} u_{j}(R)\right) .
\end{aligned}
$$

Further, by 5.6 and 5.7) we obtain

$$
\begin{aligned}
\lim _{R \rightarrow \infty} \sum_{i \in I} m_{i}(R)\left(2 \log R+\frac{1}{2} \sum_{j \in I} a_{i, j} u_{j}(R)\right)-\frac{1}{4 \pi} \Lambda_{I}(\boldsymbol{M}) \log R \\
=\lim _{R \rightarrow \infty}(\log R) \cdot \sum_{i \in I}\left(m_{i}(R)-M_{i}\right)\left(2-\frac{1}{4 \pi} \sum_{j \in I} a_{i, j} M_{j}\right)=0 .
\end{aligned}
$$


By $\left[5.8-5.12\right.$ we finally conclude, using our assumption $\Lambda_{I}(\boldsymbol{M})=0$, that

$$
\begin{aligned}
\Psi_{\mathbb{R}^{2}}(\tilde{\boldsymbol{\rho}})= & \lim _{R \rightarrow \infty} \int_{-\infty}^{\log R} \sum_{i \in I} w_{i}^{\prime} \log w_{i}^{\prime} d s \\
& +\int_{-\infty}^{\log R}\left[2 \sum_{i \in I} w_{i}-\frac{1}{4 \pi} \sum_{i, j \in I} a_{i, j} w_{i} w_{j}\right] d s-\log (2 \pi) \sum_{i \in I} M_{i} .
\end{aligned}
$$

The result follows from 5.13 and Proposition 4.1 granted 2.16, where we replace $w_{i}(s)$ by $w_{i}(s+\log R)$ for all $i$.

Next we turn to the necessity of the conditions. We assume that $\Psi_{\mathbb{R}^{2}}$ is bounded below on $\boldsymbol{\Gamma}_{\boldsymbol{M}}\left(\mathbb{R}^{2}\right)$. Fix $\hat{\boldsymbol{\rho}} \in \boldsymbol{\Gamma}_{\boldsymbol{M}}\left(\mathbb{R}^{2}\right)$ with compact support in $B_{1}$. For each $\alpha>0$, define $\hat{\boldsymbol{\rho}}_{\alpha}(x)=\alpha^{2} \hat{\boldsymbol{\rho}}(\alpha x)$. It is easy to verify that $\hat{\boldsymbol{\rho}}_{\alpha} \in \boldsymbol{\Gamma}_{\boldsymbol{M}}\left(\mathbb{R}^{2}\right)$ and that

$$
\Psi_{\mathbb{R}^{2}}\left(\hat{\boldsymbol{\rho}}_{\alpha}\right)=\Psi_{\mathbb{R}^{2}}(\hat{\boldsymbol{\rho}})+\frac{1}{4 \pi} \Lambda_{I}(\boldsymbol{M}) \log \alpha .
$$

From (5.14) we get immediately the necessity of the condition $\Lambda_{I}(\boldsymbol{M})=0$. To see why 2.16 is necessary as well, we shall use Theorem 5 with $\Omega=B_{1}$. Extend $\rho \in \Gamma_{M}(\Omega)$ by $\mathbf{0}$ onto $\mathbb{R}^{2} \backslash B_{1}$. From $(5.13), \Psi_{\mathbb{R}^{2}}(\boldsymbol{\rho})=\Psi_{\Omega}(\boldsymbol{\rho})-\log (2 \pi) \sum_{i \in I} M_{i}$, and the necessity of (2.16) follows from the analogous result in Theorem 5

Proof of Theorem 4 ii). We shall prove existence of a minimizer using the construction of an entire solution to a Liouville system in [7, Theorem 1.4]. Below is a short description of this construction. First, using our assumption 2.26 we can find a sequence $\left\{\boldsymbol{M}^{(m)}\right\}$ such that $\boldsymbol{M}^{(m)} \rightarrow \boldsymbol{M}$ and $\Lambda_{J}\left(\boldsymbol{M}^{(m)}\right)>0$ for all $J \subseteq I$. By the results of [7] it follows that for each $m$ there exists a radially symmetric and decreasing minimizer, $\boldsymbol{\rho}^{(m)}$, for $\Psi_{B_{1}}$ over $\boldsymbol{\Gamma}_{\boldsymbol{M}^{(m)}}\left(B_{1}\right)$. It was shown in the proof of [7, Theorem 1.4] that for an appropriate choice of a sequence $R^{(m)} \rightarrow \infty$, the rescaled sequence $\tilde{\boldsymbol{\rho}}^{(m)}(x)=\left(R^{(m)}\right)^{-2} \boldsymbol{\rho}^{(m)}\left(x / R^{(m)}\right)$ satisfies

$$
\lim _{m \rightarrow \infty} \tilde{\boldsymbol{\rho}}^{(m)}=\tilde{\boldsymbol{\rho}}^{(\infty)} \quad \text { locally uniformly on } \mathbb{R}^{2},
$$

with $\tilde{\rho}_{i}^{(\infty)}>0$ and $\int_{\mathbb{R}^{2}} \rho_{i}^{(\infty)}=M_{i}$ for all $i \in I$. Moreover, setting

$$
\tilde{u}_{i}^{(\infty)}(x)=-\frac{1}{2 \pi} \int_{\mathbb{R}^{2}} \tilde{\rho}_{i}^{(\infty)}(y) \log |x-y| d y, \quad \forall i \in I,
$$

we obtain an entire solution to the Liouville system

$$
-\Delta \tilde{u}_{i}^{(\infty)}=\lambda_{i}^{(\infty)} \exp \left(\sum_{j \in I} a_{i, j} \tilde{u}_{j}^{(\infty)}\right), \quad \forall i \in I, \text { on } \mathbb{R}^{2},
$$

for some positive constants $\lambda_{i}^{(\infty)}, i=1, \ldots, n$. Clearly, each $\tilde{\boldsymbol{\rho}}^{(m)}$ is a minimizer for $\Psi_{R_{R^{(m)}}}$ over $\boldsymbol{\Gamma}_{\boldsymbol{M}^{(m)}}\left(B_{\left.R^{(m)}\right)}\right.$. It will also be useful to consider, as in [7], $\tilde{v}_{i}^{(m)}=\log \tilde{\rho}_{i}^{(m)}$ for all $i \in I$, which satisfies

$$
-\Delta \tilde{v}_{i}^{(m)}=\sum_{j \in I} a_{i, j} \exp \left(\tilde{v}_{j}^{(m)}\right) \quad \text { on } B_{R^{(m)}} .
$$


We want to prove that $\rho^{(\infty)}$ is a minimizer for $\Psi_{\mathbb{R}^{2}}$ over $\boldsymbol{\Gamma}_{M}\left(\mathbb{R}^{2}\right)$. Note first that each $\rho \in \boldsymbol{\Gamma}_{\boldsymbol{M}^{(m)}}\left(B_{R^{(m)}}\right)$ can also be considered as a member in $\boldsymbol{\Gamma}_{\boldsymbol{M}^{(m)}}\left(\mathbb{R}^{2}\right)$, by extending it by zero outside $B_{R^{(m)}}$. For such $\rho$ we have (see $(5.9)$ )

$$
\Psi_{\mathbb{R}^{2}}(\boldsymbol{\rho})=\bar{\Psi}_{R^{(m)}}(\boldsymbol{\rho})=\sum_{i \in I} \int_{B_{R^{(m)}}} \rho_{i} \log \rho_{i} d x-\frac{1}{2} \sum_{i, j \in I} a_{i, j} \int_{B_{R^{(m)}}} \rho_{i} u_{j} d x,
$$

with

$$
u_{i}(x)=-\frac{1}{2 \pi} \int_{B_{R^{(m)}}} \rho_{i}(y) \log |x-y| d y, \quad \forall i \in I .
$$

Define also

$$
v_{i}(x)=\int_{\mathbb{R}^{2}} \rho_{i}(y) G_{B_{R^{(m)}}}(x, y) d y, \quad \forall i \in I .
$$

Then $u_{i}-v_{i}$ is a radial harmonic function on $B_{R^{(m)}}$, which must be identically equal to the constant

$$
\left.u_{i}\left(R^{(m)}\right)-v_{i}\left(R^{(m)}\right)=u_{i}\left(R^{(m)}\right)=-\frac{M_{i}^{(m)}}{2 \pi} \log R^{(m)} \quad \text { (using } 5.5 p\right) .
$$

Therefore, by definition of $\Psi_{B_{R^{(m)}}}$,

$$
\begin{aligned}
\Psi_{\mathbb{R}^{2}}(\boldsymbol{\rho})-\Psi_{B_{R^{(m)}}}(\boldsymbol{\rho}) & =\frac{1}{2} \sum_{i, j \in I} a_{i, j} \int_{B_{R^{(m)}}} \rho_{i}\left(v_{j}-u_{j}\right) d x \\
& =\frac{1}{4 \pi} \sum_{i, j \in I} a_{i, j} M_{i}^{(m)} M_{j}^{(m)} \log R^{(m)} .
\end{aligned}
$$

It follows from $(5.18)$ that for each $m, \tilde{\boldsymbol{\rho}}^{(m)}$ is a minimizer for $\Psi_{\mathbb{R}^{2}}$ (equivalently, of $\left.\bar{\Psi}_{R^{(m)}}\right)$ over $\boldsymbol{\Gamma}_{\boldsymbol{M}^{(m)}}\left(B_{R^{(m)}}\right)$.

Put

$$
u_{i}^{(m)}(x)=-\frac{1}{2 \pi} \int_{B_{R^{(m)}}} \tilde{\rho}_{i}^{(m)}(y) \log |x-y| d y, \quad \forall i \in I .
$$

A simple but important consequence of our assumption 2.26 is

$$
\frac{1}{4 \pi} \frac{\partial \Lambda_{I}}{\partial M_{i}}(\boldsymbol{M})=2-\frac{1}{2 \pi} \sum_{j \in I} a_{i, j} M_{j}<0, \quad \forall i \in I \quad \text { (see [7, Lemma 5.1]), }
$$

which implies the existence of $\varepsilon_{0}>0$ and $R_{0}>0$ such that

$$
\frac{1}{2 \pi} \int_{B_{R_{0}}} \sum_{j \in I} a_{i, j} \tilde{\rho}_{i}^{(\infty)}(x) d x \geq 2+2 \varepsilon_{0}, \quad \forall i \in I .
$$

Using (5.15) we deduce that, for $m_{0}$ large enough,

$$
\frac{1}{2 \pi} \int_{B_{R_{0}}} \sum_{j \in I} a_{i, j} \tilde{\rho}_{i}^{(m)}(x) d x \geq 2+\varepsilon_{0}, \quad \forall i \in I, \forall m \geq m_{0} .
$$


By (5.17) and 5.21) we obtain

$$
-\frac{\sum_{j \in I} a_{i, j} M_{j}}{2 \pi r} \leq \frac{\partial \tilde{v}_{i}^{(m)}}{\partial r}(r) \leq-\frac{2+\varepsilon_{0}}{r}, \quad \forall r \in\left[R_{0}, R^{(m)}\right], \forall i \in I
$$

An immediate consequence of 5.22 and 5.15 is that for some constants $c$ and $c_{1}$,

$$
c_{1}-\frac{\sum_{j \in I} a_{i, j} M_{j}}{2 \pi} \log r \leq \tilde{v}_{i}^{(m)}(r) \leq c-\left(2+\varepsilon_{0}\right) \log r, \quad \forall r \in\left[R_{0}, R^{(m)}\right], \quad \forall i \in I,
$$

and therefore

$$
\tilde{\rho}_{i}^{(m)}(r) \leq \frac{e^{c}}{r^{2+\varepsilon_{0}}}, \quad \forall r \geq R_{0}, \forall i \in I .
$$

From 5.24, 5.23, 5.15 and dominated convergence we obtain

$$
\lim _{m \rightarrow \infty} \Psi_{\mathbb{R}^{2}}\left(\tilde{\boldsymbol{\rho}}^{(m)}\right)=\Psi_{\mathbb{R}^{2}}\left(\tilde{\boldsymbol{\rho}}^{(\infty)}\right)
$$

Fix now any $\tilde{\boldsymbol{\rho}} \in \boldsymbol{\Gamma}_{\boldsymbol{M}}\left(\mathbb{R}^{2}\right)$. As explained in the proof of assertion (i), it is enough to consider $\tilde{\boldsymbol{\rho}}$ whose components are radially symmetric and decreasing. Fix $\alpha>0$ and $\delta>0$ such that

$$
\tilde{\rho}_{i}(x) \geq \delta, \quad \forall x \in B_{\alpha}, \forall i \in I .
$$

Let the function $\zeta$ be defined by $\zeta=\frac{1}{\pi \alpha^{2}} \chi_{B_{\alpha}}$. Define $\tilde{M}$ by setting $\tilde{M}_{i}^{(m)}=\int_{B_{R^{(m)}}} \tilde{\rho}_{i}$ for all $i \in I$, and then

$$
\hat{\boldsymbol{\rho}}^{(m)}=\chi_{B_{R^{(m)}}} \tilde{\boldsymbol{\rho}}+\zeta\left(\boldsymbol{M}^{(m)}-\tilde{\boldsymbol{M}}^{(m)}\right) .
$$

For $m$ large enough $\hat{\boldsymbol{\rho}}^{(m)} \in \boldsymbol{\Gamma}_{\boldsymbol{M}^{(m)}}\left(B_{R^{(m)}}\right)$, and since $\tilde{\boldsymbol{\rho}}^{(m)}$ is a minimizer for $\bar{\Psi}_{R^{(m)}}$ over $\boldsymbol{\Gamma}_{\boldsymbol{M}^{(m)}}\left(B_{R^{(m)}}\right)$, we have

$$
\Psi_{\mathbb{R}^{2}}\left(\tilde{\boldsymbol{\rho}}^{(m)}\right) \leq \Psi_{\mathbb{R}^{2}}\left(\hat{\boldsymbol{\rho}}^{(m)}\right) .
$$

By (5.27), dominated convergence and 5.25) we infer that

$$
\Psi_{\mathbb{R}^{2}}(\tilde{\boldsymbol{\rho}})=\lim _{m \rightarrow \infty} \Psi_{\mathbb{R}^{2}}\left(\chi_{B_{R}(m)} \tilde{\boldsymbol{\rho}}\right)=\lim _{m \rightarrow \infty} \Psi_{\mathbb{R}^{2}}\left(\hat{\boldsymbol{\rho}}^{(m)}\right) \geq \lim _{m \rightarrow \infty} \Psi_{\mathbb{R}^{2}}\left(\tilde{\boldsymbol{\rho}}^{(m)}\right)=\Psi_{\mathbb{R}^{2}}\left(\tilde{\boldsymbol{\rho}}^{(\infty)}\right),
$$

and the result follows.

Finally, the necessity of condition 2.26 for the existence of a minimizer is an immediate consequence of [7], Theorem 1.4]. Indeed, the existence of a minimizer $\tilde{\boldsymbol{\rho}}^{(\infty)}$ implies the existence of an entire solution to the Liouville system [5.16). But it was shown in [7] that 2.26 is necessary for the latter to hold.

Finally, we turn to the proof of our main result on $S^{2}$, Theorem 2 .

Proof of Theorem 2 We first remark that by the duality principle of Corollary 3.1, it is enough to prove assertion (i), which implies assertion (ii). Next we prove the sufficiency of condition 2.16. For each $i \in I$, denote by $\rho_{i}^{*}$ the symmetric decreasing rearrangement 
of $\rho_{i}$ (with respect to the north pole). Clearly $\int_{S^{2}} \rho_{i}^{*} \log \rho_{i}^{*}=\int_{S^{2}} \rho_{i} \log \rho_{i}$ for all $i$ and by a result of Baernstein and Taylor [2, Theorem 2] (see also [3, 4]) we have

$\int_{S^{2}} \int_{S^{2}} \rho_{i}(x)(\log |x-y|) \rho_{j}(y) d x d y \geq \int_{S^{2}} \int_{S^{2}} \rho_{i}^{*}(x)(\log |x-y|) \rho_{j}^{*}(y) d x d y, \quad \forall i, j$.

Therefore, we may assume that each $\rho_{i}$ is radially symmetrically decreasing from the north pole. Moreover, by a simple density argument, we may assume that the support of each $\rho_{i}$ does not intersect a certain neighborhood of the north pole. Next we use stereographic projection in order to restate the variational problem in an equivalent form on $\mathbb{R}^{2}$. More precisely, defining $\tilde{\boldsymbol{\rho}}$ by (2.4) we deduce by (2.6) that $\Psi_{S^{2}}(\boldsymbol{\rho})=\widetilde{\Psi}_{\mathbb{R}^{2}}(\tilde{\boldsymbol{\rho}})$. Moreover, our assumption on the support of $\rho$ implies that $\tilde{\rho}$ is supported in some disc $B_{R_{0}}$. Therefore, for any $R \geq R_{0}$ we have,

$$
\begin{aligned}
\widetilde{\Psi}_{\mathbb{R}^{2}}(\tilde{\boldsymbol{\rho}})= & \sum_{i \in I} \int_{B_{R}} \tilde{\rho}_{i} \log \tilde{\rho}_{i} d x+\frac{1}{4 \pi} \sum_{i, j \in I} a_{i, j} \int_{B_{R}} \int_{B_{R}} \tilde{\rho}_{i}(x)(\log |x-y|) \tilde{\rho}_{j}(y) d x d y \\
& +\sum_{i \in I} v_{i} \int_{B_{R}} \tilde{\rho}_{i} \log \left(1+|x|^{2}\right) d x .
\end{aligned}
$$

As in the proof of Theorem 4 we shall use

$$
\begin{aligned}
& u_{i}(x)=-\frac{1}{2 \pi} \int_{\mathbb{R}^{2}} \tilde{\rho}_{i}(y) \log |x-y| d y, \\
& m_{i}(r)=2 \pi \int_{0}^{r} s \rho_{i}(s) d s \quad \text { and } \quad w_{i}(s)=m_{i}\left(e^{s}\right) .
\end{aligned}
$$

From

$$
\begin{aligned}
\int_{B_{R}} \tilde{\rho}_{i} \log \left(1+|x|^{2}\right) d x & =\int_{0}^{R} m_{i}^{\prime}(r) \log \left(1+r^{2}\right) d r \\
& =M_{i} \log \left(1+R^{2}\right)-\int_{0}^{R} \frac{2 m_{i}(r) r}{1+r^{2}} d r \\
& =M_{i} \log \left(1+R^{2}\right)-\int_{-\infty}^{\log R} \frac{2 w_{i}(s) e^{2 s}}{1+e^{2 s}} d s
\end{aligned}
$$

and $5.10-5.12$ we get

$$
\begin{aligned}
\widetilde{\Psi}_{\mathbb{R}^{2}}(\tilde{\boldsymbol{\rho}})= & \int_{-\infty}^{\log R} \sum_{i \in I} w_{i}^{\prime} \log w_{i}^{\prime} d s+\int_{-\infty}^{\log R}\left[2 \sum_{i \in I} w_{i}-\frac{1}{4 \pi} \sum_{i, j \in I} a_{i, j} w_{i} w_{j}\right] d s \\
& -\sum_{i \in I}\left[2 M_{i} \log (2 \pi R)+\frac{1}{2} M_{i} \sum_{j \in I} a_{i, j} u_{j}(R)\right] \\
& +\sum_{i \in I} v_{i}\left[M_{i} \log \left(1+R^{2}\right)-\int_{-\infty}^{\log R} \frac{2 w_{i} e^{2 s}}{1+e^{2 s}} d s\right]
\end{aligned}
$$


Using (5.6) and the identity $\frac{\Lambda_{I}(\boldsymbol{M})}{4 \pi}=\sum_{i \in I} v_{i} M_{i}$ we obtain

$$
\begin{aligned}
-\sum_{i \in I}\left[2 M_{i} \log R+\frac{1}{2} M_{i} \sum_{j \in I} a_{i, j} u_{j}(R)\right] & +\sum_{i \in I} v_{i} M_{i} \log \left(1+R^{2}\right) \\
& =\left[-\frac{\Lambda_{I}(\boldsymbol{M})}{4 \pi}+2 \sum_{i \in I} v_{i} M_{i}\right] \log R+o(1) \\
& =\frac{\Lambda_{I}(\boldsymbol{M})}{4 \pi} \log R+o(1)
\end{aligned}
$$

with $o(1)$ denoting a quantity which goes to 0 as $R \rightarrow \infty$. Therefore we may write

$$
\widetilde{\Psi}_{\mathbb{R}^{2}}(\tilde{\boldsymbol{\rho}})=J_{-\infty}(\boldsymbol{w})+J_{\infty}^{R}(\boldsymbol{w})-2 \log (2 \pi) \sum_{i \in I} M_{i}+o(1)
$$

where

$$
\begin{aligned}
J_{-\infty}(\boldsymbol{w})= & \int_{-\infty}^{0} \sum_{i \in I} w_{i}^{\prime} \log w_{i}^{\prime} d s+\int_{-\infty}^{0}\left[2 \sum_{i \in I} w_{i}-\frac{1}{4 \pi} \sum_{i, j \in I} a_{i, j} w_{i} w_{j}\right] d s \\
& -\sum_{i \in I} v_{i} \int_{-\infty}^{0} \frac{2 w_{i} e^{2 s}}{1+e^{2 s}} d s,
\end{aligned}
$$

and

$$
\begin{aligned}
J_{\infty}^{R}(\boldsymbol{w})= & \int_{0}^{\log R} \sum_{i \in I} w_{i}^{\prime} \log w_{i}^{\prime} d s \\
& +\int_{0}^{\log R}\left[2 \sum_{i \in I} w_{i}-\frac{1}{4 \pi} \sum_{i, j \in I} a_{i, j} w_{i} w_{j}+\frac{\Lambda_{I}(\boldsymbol{M})}{4 \pi}\right] d s \\
& -\sum_{i \in I} v_{i} \int_{0}^{\log R} \frac{2 w_{i} e^{2 s}}{1+e^{2 s}} d s .
\end{aligned}
$$

Since clearly,

$$
\int_{-\infty}^{0} \frac{2 w_{i} e^{2 s}}{1+e^{2 s}} \leq 2 M_{i} \int_{-\infty}^{0} e^{2 s} d s=M_{i}
$$

it follows from Proposition 4.1 that $J_{-\infty}(\boldsymbol{w}) \geq-C$ for some constant $C$. Hence it remains to find a lower bound for $J_{\infty}^{R}(w)$, uniformly in $R \in\left[R_{0}, \infty\right)$.

Since

$$
\begin{aligned}
-\sum_{i \in I} v_{i} \int_{0}^{\log R} \frac{2 w_{i} e^{2 s}}{1+e^{2 s}} d s & =-2 v_{i} \sum_{i \in I} \int_{0}^{\log R} w_{i} d s+\sum_{i \in I} v_{i} \int_{0}^{\log R} \frac{2 w_{i}}{1+e^{2 s}} d s \\
& \geq-2 v_{i} \sum_{i \in I} \int_{0}^{\log R} w_{i} d s-\sum_{i \in I}\left|v_{i}\right| M_{i},
\end{aligned}
$$


it suffices to prove that the functional

$$
\begin{aligned}
G_{\infty}^{R}(\boldsymbol{w}):= & \int_{0}^{\log R} \sum_{i \in I} w_{i}^{\prime} \log w_{i}^{\prime} d s \\
& +\int_{0}^{\log R}\left[\sum_{i \in I} 2\left(1-v_{i}\right) w_{i}-\frac{1}{4 \pi} \sum_{i, j \in I} a_{i, j} w_{i} w_{j}+\frac{\Lambda_{I}(\boldsymbol{M})}{4 \pi}\right] d s
\end{aligned}
$$

is bounded below. A simple computation shows that

$$
\begin{aligned}
\sum_{i \in I} 2\left(1-v_{i}\right) w_{i}-\frac{1}{4 \pi} \sum_{i, j \in I} a_{i, j} w_{i} w_{j}+\frac{\Lambda_{I}(\boldsymbol{M})}{4 \pi} & \\
& =\sum_{i \in I} 2\left(M_{i}-w_{i}\right)-\frac{1}{4 \pi} \sum_{i, j \in I} a_{i, j}\left(M_{i}-w_{i}\right)\left(M_{j}-w_{j}\right) .
\end{aligned}
$$

Therefore, setting for each $i \in I, z_{i}(t)=M_{i}-w_{i}(-t)$ for $t \in[-\log R, 0]$ and $z_{i}(t)=0$ for $t \in(-\infty,-\log R)$, we infer from (5.33) that

$$
G_{\infty}^{R}(\boldsymbol{w})=\int_{-\infty}^{0}\left[\sum_{i \in I} z_{i}^{\prime} \log z_{i}^{\prime}+\frac{1}{4 \pi} \Lambda_{I}(z)\right] d t
$$

Since $z_{i}(-\infty)=0$ and $z_{i}(0)=M_{i}-w_{i}(0) \leq M_{i}$ for all $i$, we can apply Proposition 4.1 to conclude that the r.h.s. of (5.34) is bounded from below, completing the proof of the sufficiency assertion.

For the proof of necessity of 2.16$)$, we consider $\widetilde{\Psi}_{\mathbb{R}^{2}}(\tilde{\boldsymbol{\rho}})$ for $\tilde{\boldsymbol{\rho}}$ with support in $B_{1}$. Since $J_{\infty}^{1}(\boldsymbol{w})=0$ for the corresponding $\boldsymbol{w}$, we conclude from 5.30 that $\widetilde{\Psi}_{\mathbb{R}^{2}}$ is bounded below on the class of such $\tilde{\rho}$ 's iff $J_{-\infty}$ is bounded below on the corresponding class of $\boldsymbol{w}$ 's. But the necessity assertion of Proposition 4.1 implies that 2.16 is necessary for the latter to hold.

\subsection{Multi-block systems}

We start with the proof of assertion (i) of Theorem 3 , dealing with a general collaborating system with $K$ blocks.

Proof of Theorem 3 (i). (i) Setting for each $l=1, \ldots, K$,

$$
\Psi_{S^{2}}^{\left(I_{l}\right)}(\boldsymbol{\rho})=\sum_{i \in I_{l}} \int_{S^{2}} \rho_{i} \log \rho_{i}+\frac{1}{4 \pi} \sum_{i, j \in I_{l}} a_{i, j} \int_{S^{2}} \int_{S^{2}} \rho_{i}(x)(\log |x-y|) \rho_{j}(y) d x d y,
$$

we may write

$\Psi_{S^{2}}(\boldsymbol{\rho})=\sum_{l=1}^{K} \Psi_{S^{2}}^{\left(I_{l}\right)}(\boldsymbol{\rho})+\frac{1}{4 \pi} \sum_{l_{1} \neq l_{2}} \sum_{i \in I_{l_{1}}} \sum_{j \in I_{l_{2}}} a_{i, j} \int_{S^{2}} \int_{S^{2}} \rho_{i}(x)(\log |x-y|) \rho_{j}(y) d x d y$. 
Using the inequality $|x-y| \leq 2$ for all $x, y \in S^{2}$, and the assumption 2.17), we get

$$
\begin{aligned}
\sum_{l_{1} \neq l_{2}} \sum_{i \in I_{l_{1}}} \sum_{j \in I_{l_{2}}} a_{i, j} \int_{S^{2}} \int_{S^{2}} \rho_{i}(x)(\log |x-y|) \rho_{j}(y) d x d y & \\
& \geq(\log 2) \cdot \sum_{l_{1} \neq l_{2}} \sum_{i \in I_{l_{1}}} \sum_{j \in I_{l_{2}}} a_{i, j} M_{i} M_{j} .
\end{aligned}
$$

Therefore, if condition 2.21) is satisfied, then from Theorem2 it follows that

$$
\Psi_{S^{2}}^{\left(I_{l}\right)}(\boldsymbol{\rho}) \geq-C, \quad \forall \boldsymbol{\rho} \in \boldsymbol{\Gamma}_{\boldsymbol{M}}\left(S^{2}\right), l=1, \ldots, K,
$$

and by 5.36) and 5.37) we obtain

$$
\Psi_{S^{2}}(\boldsymbol{\rho}) \geq-C, \quad \forall \boldsymbol{\rho} \in \boldsymbol{\Gamma}_{\boldsymbol{M}}\left(S^{2}\right) .
$$

To prove the necessity of condition 2.21, assume by negation that for some $1 \leq l \leq K$, condition 2.21 is violated. Then by Theorem 2 there exists a sequence

$$
\left\{\boldsymbol{\rho}^{m}=\left(\rho_{i}^{m}\right)_{i \in I_{l}}\right\} \subset \boldsymbol{\Gamma}_{\boldsymbol{M}^{(l)}}\left(S^{2}\right),
$$

with $\boldsymbol{\Gamma}_{\boldsymbol{M}^{(l)}}\left(S^{2}\right)$ denoting the restriction of $\boldsymbol{\Gamma}_{\boldsymbol{M}}\left(S^{2}\right)$ to the coordinates of $I_{l}$, such that $\lim _{m \rightarrow \infty} \Psi_{S^{2}}^{\left(I_{l}\right)}\left(\boldsymbol{\rho}^{m}\right)=-\infty$. Extend each $\boldsymbol{\rho}^{m}$ to $\boldsymbol{\Gamma}_{\boldsymbol{M}}\left(S^{2}\right)$ by setting

$$
\rho_{i}^{m}(x)= \begin{cases}\rho_{i}^{m}(x) & \text { if } i \in I_{l}, \\ M_{i} f(x) & \text { if } i \notin I_{l},\end{cases}
$$

where $f$ is a smooth positive function on $S^{2}$ with $\int_{S^{2}} f(x) d x=1$. Then it is easy to verify, as in the proof of the necessity part of Theorem 2, that $\lim _{m \rightarrow \infty} \Psi_{S^{2}}\left(\boldsymbol{\rho}^{m}\right)=-\infty$.

We next give the proof of assertion (ii) of Theorem 5 which deals with the multi-block case for a system on a bounded domain.

Proof of Theorem 5 ii). The proof uses the same argument as in Theorem $3(\mathrm{i})$. It suffices to note that $G_{\Omega}(x, y)=-\frac{1}{2 \pi} \log |x-y|+R_{y}(x)$ with $R_{y}(x)$ a harmonic function on $\Omega$, which is bounded above by $\frac{1}{2 \pi} \log (\operatorname{diam}(\Omega))$ for all $y \in \Omega$.

For the proof of assertion (ii) of Theorem 3 we shall need the following lemma. For a symmetric $n$ by $n$ matrix $A$ satisfying $a_{i, j} \leq 0$ for all $i \neq j$ (here the diagonal elements play no role) consider the functional

$$
J(\boldsymbol{\mu})=\sum_{i \neq j}\left(-a_{i, j}\right) \int_{S^{2}} \int_{S^{2}} \log |x-y| d \mu_{i}(x) d \mu_{j}(y),
$$

defined over the following set of $n$-vectors of finite Borel measures:

$$
\mathcal{G}_{\boldsymbol{M}}\left(S^{2}\right)=\left\{\boldsymbol{\mu}=\left(\mu_{1}, \ldots, \mu_{n}\right): \mu_{i} \geq 0 \text { and } \int_{S^{2}} d \mu_{i}=M_{i}, \forall i\right\} .
$$

Note that $J$ is well defined on $\mathcal{G}_{\boldsymbol{M}}\left(S^{2}\right)$ if we allow it to take the value $-\infty$, since the kernel $\log |x-y|$ is bounded above and $a_{i, j} \leq 0$ for $i \neq j$. 
Lemma 5.1. Let $A$ be a symmetric matrix with $a_{i, j} \leq 0$ for all $i \neq j$ and let $\boldsymbol{M} \in \mathbb{R}_{+}^{n}$. Then

$$
\sup _{\boldsymbol{\mu} \in \mathcal{G}_{\boldsymbol{M}}\left(S^{2}\right)} J(\boldsymbol{\mu})=\max _{\boldsymbol{y} \in\left(S^{2}\right)^{n}} W(\boldsymbol{y}) \quad(\text { see } 2.23),
$$

and the supremum is attained at measures of the form

$$
\boldsymbol{\mu}=\left(M_{1} \delta_{x_{1}}, \ldots, M_{n} \delta_{x_{n}}\right), \text { with } \boldsymbol{x}=\left(x_{1}, \ldots, x_{n}\right) \in\left(S^{2}\right)^{n} \text { a maximizer of } W .
$$

Moreover, if A does not contain a row of zeros (ignoring the diagonal elements), then all the maxima of $J$ and all the weak limits, in the sense of measures, of maximizing sequences are of the form 5.41.

Proof. Consider any $\boldsymbol{\mu} \in \mathcal{G}_{M}\left(S^{2}\right)$. For each $i \in I$ set

$$
U_{i}^{\mu}(x)=2 \sum_{j \neq i}\left(-a_{i, j}\right) \int_{S^{2}} \log |x-y| d \mu_{j}(y) .
$$

Then we have

$$
J(\boldsymbol{\mu})=\sum_{\{i \neq j \neq k \neq i\}}\left(-a_{j, k}\right) \int_{S^{2}} \int_{S^{2}} \log |x-y| d \mu_{j}(x) d \mu_{k}(y)+\int_{S^{2}} U_{i}^{\mu}(x) d \mu_{i}(x) .
$$

It is known that $U_{i}^{\mu}$ is upper semicontinuous (see [17]), and therefore its maximum on $S^{2}$ is attained. For any $i \in I$ define $T_{i, y_{i}}(\boldsymbol{\mu}) \in \mathcal{G}_{M}\left(S^{2}\right)$ by

$$
\left(T_{i, y_{i}}(\boldsymbol{\mu})\right)_{j}= \begin{cases}\mu_{j} & \text { for } j \neq i, \\ M_{i} \delta_{y_{i}} & \text { for } j=i,\end{cases}
$$

where $y_{i} \in S^{2}$ is any maximum point of $U_{i}^{\mu}$. It is clear that $J\left(T_{i, y_{i}}(\boldsymbol{\mu})\right) \geq J(\boldsymbol{\mu})$. Setting

$$
\overline{\boldsymbol{\mu}}=T_{n, y_{n}} \circ T_{n-1, y_{n-1}} \circ \cdots \circ T_{1, y_{1}}(\boldsymbol{\mu})=\left(M_{1} \delta_{y_{1}}, \ldots, M_{n} \delta_{y_{n}}\right),
$$

we have

$$
W(\boldsymbol{y})=J(\overline{\boldsymbol{\mu}}) \geq J(\boldsymbol{\mu})
$$

and 5.40 follows.

To prove the last claim, let $\boldsymbol{\mu}$ be a maximizer for $J$ over $\mathcal{G}_{M}\left(S^{2}\right)$. Assume by negation that for one of the components of $\boldsymbol{\mu}$, say $\mu_{1}$, $\operatorname{supp}\left(\mu_{1}\right)$ is not a singleton. Consider then

$$
\overline{\boldsymbol{\mu}}=T_{n, y_{n}} \circ T_{n-1, y_{n-1}} \circ \cdots \circ T_{2, y_{2}}(\boldsymbol{\mu})=\left(\mu_{1}, M_{2} \delta_{y_{2}}, \ldots, M_{n} \delta_{y_{n}}\right) .
$$

By construction, $\bar{\mu}$ is also a maximizer, for which $U_{1}^{\bar{\mu}}$ must be constant on $\operatorname{supp}\left(\mu_{1}\right)$ (otherwise, we would have $J\left(T_{1, y_{1}}(\bar{\mu})\right)>J(\bar{\mu})$ for $y_{1}$ a maximum point of $U_{1}^{\bar{\mu}}$ ). For two distinct points $x_{1}, z_{1} \in \operatorname{supp}\left(\mu_{1}\right)$ put

$$
\begin{aligned}
& \boldsymbol{\mu}_{x_{1}}=T_{1, x_{1}}(\overline{\boldsymbol{\mu}})=\left(M_{1} \delta_{x_{1}}, M_{2} \delta_{y_{2}}, \ldots, M_{n} \delta_{y_{n}}\right), \\
& \boldsymbol{\mu}_{z_{1}}=T_{1, z_{1}}(\overline{\boldsymbol{\mu}})=\left(M_{1} \delta_{z_{1}}, M_{2} \delta_{y_{2}}, \ldots, M_{n} \delta_{y_{n}}\right) .
\end{aligned}
$$


Then also $\boldsymbol{\mu}_{x_{1}}$ and $\boldsymbol{\mu}_{z_{1}}$ are maximizers for $J$, i.e. $\left(x_{1}, y_{2}, \ldots, y_{n}\right)$ and $\left(z_{1}, y_{2}, \ldots, y_{n}\right)$ are maximizers for $W$. We must then have for each $j \neq 1$,

$$
\frac{\partial W}{\partial y_{j}}\left(x_{1}, y_{2}, \ldots, y_{n}\right) \times y_{j}=\frac{\partial W}{\partial y_{j}}\left(z_{1}, y_{2}, \ldots, y_{n}\right) \times y_{j}=0,
$$

i.e.

$$
\begin{aligned}
0 & =\left(-a_{1, j}\right) M_{1} M_{j} \frac{\left(y_{j}-x_{1}\right) \times y_{j}}{\left|y_{j}-x_{1}\right|^{2}}+\sum_{i \neq j, 1}\left(-a_{i, j}\right) M_{i} M_{j} \frac{\left(y_{j}-y_{i}\right) \times y_{j}}{\left|y_{j}-y_{i}\right|^{2}} \\
& =\left(-a_{1, j}\right) M_{1} M_{j} \frac{\left(y_{j}-z_{1}\right) \times y_{j}}{\left|y_{j}-z_{1}\right|^{2}}+\sum_{i \neq j, 1}\left(-a_{i, j}\right) M_{i} M_{j} \frac{\left(y_{j}-y_{i}\right) \times y_{j}}{\left|y_{j}-y_{i}\right|^{2}} .
\end{aligned}
$$

By assumption, there exists $j_{0} \neq 1$ with $a_{1, j_{0}}<0$. For this $j_{0}$ we deduce from 5.44 that

$$
\frac{\left(y_{j_{0}}-x_{1}\right) \times y_{j_{0}}}{\left|y_{j_{0}}-x_{1}\right|^{2}}=\frac{\left(y_{j_{0}}-z_{1}\right) \times y_{j_{0}}}{\left|y_{j_{0}}-z_{1}\right|^{2}} \text {. }
$$

This last equality forces $x_{1}=z_{1}$. Contradiction. Finally, the statement about the weak limits of maximizing sequences follows from the upper semicontinuity with respect to weak convergence of measures and the characterization of the maxima.

Proof of Theorem 3 completed. (ii) Since $K=n$, 5.35) takes the form

$\Psi_{S^{2}}^{\left(I_{l}\right)}(\boldsymbol{\rho})=\psi_{l}\left(\rho_{l}\right):=\int_{S^{2}} \rho_{l} \log \rho_{l}+\frac{a_{l, l}}{4 \pi} \int_{S^{2}} \int_{S^{2}} \rho_{l}(x)(\log |x-y|) \rho_{l}(y) d x d y, \quad \forall l \in I$.

Here, in the critical case of $a_{i, i} M_{i}=8 \pi$ for all $i$, by Theorem 2 (see [3.10p) we have

$$
\psi_{i}\left(\rho_{i}\right) \geq \frac{M_{i}}{2 \pi} c_{0}+M_{i} \log \frac{M_{i}}{4 \pi}, \quad \forall i \in I .
$$

Moreover, by Lemma 5.1 .

$$
\frac{1}{4 \pi} \sum_{i \neq j} a_{i, j} \int_{S^{2}} \int_{S^{2}} \rho_{i}(x)(\log |x-y|) \rho_{j}(y) d x d y \geq-\frac{1}{4 \pi} \sup _{\left(S^{2}\right)^{n}} W(x) .
$$

Plugging 5.45-5.46, in 5.36, we are led to

$$
\inf _{\Gamma_{M}\left(S^{2}\right)} \Psi_{S^{2}} \geq \sum_{i \in I}\left[M_{i} \log \frac{M_{i}}{4 \pi}+\frac{M_{i}}{2 \pi} c_{0}\right]-\frac{1}{4 \pi} \sup _{\left(S^{2}\right)^{n}} W(x) .
$$

Let $z \in\left(S^{2}\right)^{n}$ be a maximizer for $W$. For each $\alpha>0$ define $\rho^{(\alpha)}=\left(\rho^{0}\right)^{\tau_{x_{i}, \alpha}}$ applying 2.2) componentwise with $\rho^{0}=\left(M_{1} / 4 \pi, \ldots, M_{n} / 4 \pi\right)$. By Theorem 1 , for each $i \in I$, $\rho_{i}^{(\alpha)}$ gives equality in 5.45). Combining it with

$$
\lim _{\alpha \rightarrow \infty}-\sum_{i \neq j} a_{i, j} \int_{S^{2}} \int_{S^{2}} \rho_{i}^{(\alpha)}(x)(\log |x-y|) \rho_{j}^{(\alpha)}(y) d x d y=W(z)
$$


we are led to

$$
\lim _{\alpha \rightarrow \infty} \Psi_{S^{2}}\left(\rho^{(\alpha)}\right)=\sum_{i \in I}\left[M_{i} \log \frac{M_{i}}{4 \pi}+\frac{M_{i}}{2 \pi} c_{0}\right]-\frac{1}{4 \pi} \sup _{\left(S^{2}\right)^{n}} W(\boldsymbol{x}) .
$$

This together with 5.47) leads to 2.22.

(iii) Suppose by negation that there exists $\rho \in \boldsymbol{\Gamma}_{\boldsymbol{M}}\left(S^{2}\right)$ which realizes the infimum in (2.22). It follows from the above that for each $i \in I, \rho_{i}$ is a minimizer of $\psi_{i}$ over $\Gamma_{M_{i}}\left(S^{2}\right)$, and that $\boldsymbol{\mu}=\left(\rho_{1} d x, \ldots, \rho_{n} d x\right)$ is a maximizer for $J$ over $\mathcal{G}_{M}\left(S^{2}\right)$ (see (5.38), (5.39). But this contradicts the description of the maxima of $J$ given by Lemma 5.1. The statement about the weak limits of minimizing sequences follows similarly.

Remark 5.1. For the special case of the Toda system with $A$ given by (2.19), the critical case is $M_{i}=4 \pi$ for all $i \in I$. Here we find

$$
W(\boldsymbol{x})=2 \sum_{i=1}^{n-1} \log \left|x_{i+1}-x_{i}\right|,
$$

which achieves its maximum only at configurations of the form

$$
x_{j}= \begin{cases}y_{1} & \text { if } j \text { is odd } \\ y_{2} & \text { if } j \text { is even }\end{cases}
$$

where $y_{1}$ and $y_{2}$ are antipodal points in $S^{2}$.

We now present a generalization of Theorem 3(ii),(iii) to a larger class of systems than 2.18. Consider a symmetric matrix $A$ with the following properties:

(P1) The set $I$ is a disjoint union of sets $I_{1}, \ldots, I_{K}$ where each submatrix $A\left[I_{l}, I_{l}\right]$ is a conformal block, that is, for each $l=1, \ldots, K$,

$$
a_{i, j} \geq 0, \quad \forall i, j \in I_{l} \quad \text { and } \quad \sum_{j \in I_{l}} a_{i, j} M_{j}=8 \pi, \quad \forall i \in I_{l} .
$$

(P2) If $l \neq m$ then $a_{i, j}=a_{j, i} \leq 0$ for all $i \in I_{l}, j \in I_{m}$.

Let us define a "renormalization" of the system in the following sense. We define $\widehat{\boldsymbol{M}} \in \mathbb{R}_{+}^{K}$ by

$$
\widehat{M}_{l}=\sum_{i \in I_{l}} M_{i}, \quad \forall l=1, \ldots, K
$$

and $\widehat{A}=\left\{\widehat{a}_{l, m}\right\}, l, m=1, \ldots, K$, by

$$
\widehat{a}_{l, m}=\frac{\sum_{i \in I_{l}, j \in I_{m}} a_{i, j} M_{i} M_{j}}{\widehat{M}_{l} \widehat{M}_{m}} .
$$

The associated function $\widehat{W}$ is defined by

$$
\widehat{W}(\widehat{\boldsymbol{x}}):=\sum_{l \neq m}\left(-\widehat{a}_{l, m}\right) \widehat{M}_{l} \widehat{M}_{m} \log \left|\widehat{x}_{l}-\widehat{x}_{m}\right|, \quad \forall \widehat{\boldsymbol{x}} \in\left(S^{2}\right)^{K} .
$$


The new problem is to minimize the functional $\widehat{\Psi}_{S^{2}}$, associated with $\widehat{A}$, over $\boldsymbol{\Gamma}_{\widehat{\boldsymbol{M}}}\left(S^{2}\right)$. Note that for each $1 \leq l \leq K$ we have, by condition (P1),

$$
\widehat{a}_{l, l} \widehat{M}_{l}=\left(\sum_{i, j \in I_{l}} a_{i, j} M_{i} M_{j}\right) / \widehat{M}_{l}=8 \pi .
$$

Therefore, the conditions of Theorem 3 (ii) are satisfied by the new system. We do not know however whether results analogous to Theorem 3 (ii),(iii) hold, in general, for the original problem of minimizing $\Psi_{S^{2}}$ over $\boldsymbol{\Gamma}_{M}\left(S^{2}\right)$. We were only able to handle two special cases given below. The first case is $K=2$. Note that in this case, for each $\widehat{\boldsymbol{x}}=$ $\left(\widehat{x}_{1}, \widehat{x}_{2}\right) \in\left(S^{2}\right)^{2}$ we have

$$
\widehat{W}(\widehat{\boldsymbol{x}})=2\left(\log \left|\widehat{x}_{1}-\widehat{x}_{2}\right|\right) \sum_{i \in I_{1}, j \in I_{2}}\left(-a_{i, j}\right) M_{i} M_{j}
$$

Therefore,

$$
\sup _{\widehat{\boldsymbol{x}} \in\left(S^{2}\right)^{2}} \widehat{W}(\widehat{\boldsymbol{x}})=2(\log 2) \sum_{i \in I_{1}, j \in I_{2}}\left(-a_{i, j}\right) M_{i} M_{j}
$$

Proposition 5.1. Assume $A$ and $M$ are such that conditions $(\mathrm{P} 1)-(\mathrm{P} 2)$ are satisfied with $K=2$. Then

$$
\inf _{\Gamma_{M}\left(S^{2}\right)} \Psi_{S^{2}}=\sum_{l=1}^{2} \sum_{i \in I_{l}}\left[M_{i} \log \frac{M_{i}}{4 \pi}+\frac{M_{i}}{2 \pi} c_{0}\right]-\frac{\log 2}{2 \pi} \sum_{i \in I_{1}, j \in I_{2}}\left(-a_{i, j}\right) M_{i} M_{j} .
$$

If, in addition, there exist $i_{1} \in I_{1}$ and $j_{1} \in I_{2}$ with $a_{i_{1}, j_{1}} \neq 0$, then the infimum in 5.53) is not attained and any weak limit $\boldsymbol{\mu}$ of a minimizing sequence is of the form

$$
\mu_{i}= \begin{cases}M_{i} \delta_{\widehat{x}_{1}} & \text { if } i \in I_{1} \\ M_{i} \delta_{\widehat{x}_{2}} & \text { if } i \in I_{2}\end{cases}
$$

where $\widehat{x}_{1}$ and $\widehat{x}_{2}$ are antipodal points.

Remark 5.2. The Toda system, which was already seen to be a special case of Theorem 3. is also a special case of Proposition 5.1. Indeed, we can write $I=I_{1} \cup I_{2}$ with $I_{1}$ and $I_{2}$ the even and odd indices respectively.

For the proof we shall need the following lemma.

Lemma 5.2. Let $A$ be an irreducible symmetric $n$ by $n$ matrix satisfying 2.1), and let $\boldsymbol{M} \in \mathbb{R}_{+}^{n}$ be such that (2.11) holds. Suppose that $\boldsymbol{\rho}^{(m)}$ is a minimizing sequence for $\inf \Psi_{S^{2}}$ over $\boldsymbol{\Gamma}_{\boldsymbol{M}}\left(S^{2}\right)$ such that $\boldsymbol{\rho}^{(m)}-\boldsymbol{\mu}$ weakly in the sense of measures. Then $M_{j} \mu_{i}=M_{i} \mu_{j}$ for all $i, j$. 
Proof. We use a similar argument to the one used in the proof of Theorem 1 . By 2.11, we may write, for any $\rho \in \Gamma_{M}\left(S^{2}\right)$,

$$
\begin{aligned}
\Psi_{S^{2}}(\boldsymbol{\rho})=\sum_{i, j} \frac{a_{i, j}}{16 \pi}\left[M_{j} \int_{S^{2}} \rho_{i} \log \rho_{i}\right. & \\
& \left.\quad+M_{i} \int_{S^{2}} \rho_{j} \log \rho_{j}+4 \int_{S^{2}} \int_{S^{2}} \rho_{i}(x)(\log |x-y|) \rho_{j}(y) d x d y\right] .
\end{aligned}
$$

By 3.10 it follows that

$$
\begin{aligned}
\lim _{m \rightarrow \infty} & {\left[M_{j} \int_{S^{2}} \rho_{i}^{(m)} \log \rho_{i}^{(m)}+M_{i} \int_{S^{2}} \rho_{j}^{(m)} \log \rho_{j}^{(m)}\right.} \\
& \left.+4 \int_{S^{2}} \int_{S^{2}} \rho_{i}^{(m)}(x)(\log |x-y|) \rho_{j}^{(m)}(y) d x d y\right]=\frac{M_{i} M_{j}}{\pi} c_{0}+M_{i} M_{j} \log \frac{M_{i} M_{j}}{16 \pi^{2}}
\end{aligned}
$$

for all $i, j$ such that $a_{i, j}>0$. Fixing any pair of such $i, j$ and defining $F_{k}^{(m)}=\frac{4 \pi}{M_{k}} \rho_{k}^{(m)}$ for all $k$ (so that $\int_{S^{2}} F_{k}^{(m)}=4 \pi$ ) we conclude that

$$
\begin{aligned}
\lim _{m \rightarrow \infty}\left[\int_{S^{2}} F_{i}^{(m)}\right. & \log F_{i}^{(m)}+\int_{S^{2}} F_{j}^{(m)} \log F_{j}^{(m)} \\
& \left.+\frac{1}{\pi} \int_{S^{2}} \int_{S^{2}} F_{i}^{(m)}(x)(\log |x-y|) F_{j}^{(m)}(y) d x d y\right]=4 c_{0} .
\end{aligned}
$$

Note that by 3.9 we have

$$
\begin{aligned}
\widetilde{\psi}\left(F_{k}^{(m)}\right) & :=\int_{S^{2}} F_{k}^{(m)} \log F_{k}^{(m)}+\frac{1}{2 \pi} \int_{S^{2}} \int_{S^{2}} F_{k}^{(m)}(x)(\log |x-y|) F_{k}^{(m)}(y) d x d y \\
& \geq 2 c_{0}, \quad \forall k .
\end{aligned}
$$

Since

$$
\int_{S^{2}} \int_{S^{2}}(f(x)-1)(\log |x-y|)(f(y)-1) d x d y \leq 0, \quad \forall f \in \Gamma_{4 \pi}\left(S^{2}\right),
$$

we deduce that

$$
\begin{aligned}
\widetilde{\psi}\left(F_{i}^{(m)}\right)+\widetilde{\psi}\left(F_{j}^{(m)}\right) \leq & \int_{S^{2}} F_{i}^{(m)} \log F_{i}^{(m)}+\int_{S^{2}} F_{j}^{(m)} \log F_{j}^{(m)} \\
& +\frac{1}{\pi} \int_{S^{2}} \int_{S^{2}} F_{i}^{(m)}(x)(\log |x-y|) F_{j}^{(m)}(y) d x d y .
\end{aligned}
$$

Combining 5.56 with 5.54 and 5.55 we are led to

$$
\lim _{m \rightarrow \infty} \tilde{\psi}\left(F_{i}^{(m)}\right)=\lim _{m \rightarrow \infty} \tilde{\psi}\left(F_{j}^{(m)}\right)=2 c_{0} .
$$


Plugging 5.57) in 5.54) yields

$$
\begin{aligned}
& \lim _{m \rightarrow \infty}\left\{\int_{S^{2}} \int_{S^{2}} 2 F_{i}^{(m)}(x)(\log |x-y|) F_{j}^{(m)}(y) d x d y\right. \\
& \left.-\int_{S^{2}} \int_{S^{2}}\left(F_{i}^{(m)}(x)(\log |x-y|) F_{i}^{(m)}(y)+F_{j}^{(m)}(x)(\log |x-y|) F_{j}^{(m)}(y)\right) d x d y\right\}=0 .
\end{aligned}
$$

But setting

$$
U_{k}^{(m)}(x)=-\frac{1}{2 \pi} \int_{S^{2}} F_{k}^{(m)}(y) \log |x-y| d y, \quad \forall k,
$$

we may rewrite 5.58 as

$$
\lim _{m \rightarrow \infty} \int_{S^{2}}\left|\nabla\left(U_{i}^{(m)}-U_{j}^{(m)}\right)\right|^{2}=0
$$

Therefore, for every $\varphi \in C^{\infty}\left(S^{2}\right)$ we have

$$
\begin{aligned}
\frac{4 \pi}{M_{i}} \int_{S^{2}} \varphi d \mu_{i}-\frac{4 \pi}{M_{j}} \int_{S^{2}} \varphi d \mu_{j} & =\lim _{m \rightarrow \infty} \int_{S^{2}}\left(F_{i}^{(m)}-F_{j}^{(m)}\right) \varphi d x \\
& =\lim _{m \rightarrow \infty} \int_{S^{2}} \nabla\left(U_{i}^{(m)}-U_{j}^{(m)}\right) \cdot \nabla \varphi=0,
\end{aligned}
$$

i.e. $M_{j} \mu_{i}=M_{i} \mu_{j}$. The result follows by the irreducibility of $A$.

Proof of Proposition 5.1. By Theorem 1. applied to each $\Psi_{S^{2}}^{\left(I_{l}\right)}$ (see (5.35)), we have

$$
\Psi_{S^{2}}^{\left(I_{l}\right)}(\boldsymbol{\rho}) \geq \sum_{i \in I_{l}} \frac{c_{0}}{2 \pi} M_{i}+M_{i} \log \frac{M_{i}}{4 \pi}, \quad \forall l .
$$

Further, by 5.52

$$
\begin{aligned}
2 \sum_{i \in I_{1}, j \in I_{2}}\left(-a_{i, j}\right) \int_{S^{2}} \int_{S^{2}} \rho_{i}(x)(\log |x-y|) \rho_{j}(y) d x d y & \leq 2(\log 2) \sum_{i \in I_{1}, j \in I_{2}}\left(-a_{i, j}\right) M_{i} M_{j} \\
& =\sup _{\widehat{\boldsymbol{x}} \in\left(S^{2}\right)^{2}} \widehat{W}(\widehat{\boldsymbol{x}}) .
\end{aligned}
$$

If $a_{i, j}=0$ for all $i \in I_{1}$ and $j \in I_{2}$ then $\Psi_{S^{2}}=\Psi_{S^{2}}^{\left(I_{1}\right)}+\Psi_{S^{2}}^{\left(I_{2}\right)}$ and the result follows from 5.59) and Theorem 11 Assume now that at least one of these $a_{i, j}$ 's is nonzero. Then the inequality in 5.60) is strict and combining it with 5.59] we obtain, for all $\rho \in \Gamma_{M}\left(S^{2}\right)$,

$$
\Psi_{S^{2}}(\boldsymbol{\rho})>\sum_{l=1}^{2} \sum_{i \in I_{l}}\left[M_{i} \log \frac{M_{i}}{4 \pi}+\frac{M_{i}}{2 \pi} c_{0}\right]-\frac{1}{4 \pi} \sup _{\widehat{x} \in\left(S^{2}\right)^{2}} \widehat{W}(\widehat{\boldsymbol{x}}) .
$$


To finish the proof of 5.53) it is enough to show that $\Psi_{S^{2}}(\rho)$ can get as close as we wish to the value of the r.h.s. of 5.61. To this end, we fix a pair $\widehat{x}_{1}, \widehat{x}_{2} \in S^{2}$ of antipodal points, and for every $\alpha>0$ define $\rho^{(\alpha)} \in \Gamma_{M}\left(S^{2}\right)$ as follows:

$$
\rho_{i}^{(\alpha)}=\left(\rho_{i}^{0}\right)^{\tau_{\widehat{x}_{m}, \alpha}}, \quad \forall i \in I_{m}, m=1,2
$$

It is easy to see that the $\operatorname{limit}_{\alpha \rightarrow \infty} \Psi_{S^{2}}\left(\rho^{(\alpha)}\right)$ equals the r.h.s. of 5.61) and the proof of 5.53 is complete.

Next we turn to the proof of the statement about the weak limits of minimizing sequences. Let $\left\{\boldsymbol{\rho}^{(m)}\right\}$ be such a sequence with $\boldsymbol{\rho}^{(m)} \rightarrow \boldsymbol{\mu}$ weakly in the sense of measures. By the above,

$$
\lim _{m \rightarrow \infty} \Psi_{S^{2}}^{\left(I_{l}\right)}\left(\boldsymbol{\rho}^{(m)}\right)=\sum_{i \in I_{l}} \frac{c_{0}}{2 \pi} M_{i}+M_{i} \log \frac{M_{i}}{4 \pi}, \quad l=1,2,
$$

i.e. the restriction of $\rho^{(m)}$ to the indices of $I_{l}, l=1,2$, is a minimizing sequence for $\Psi_{S^{2}}^{\left(I_{l}\right)}$ over $\boldsymbol{\Gamma}_{\boldsymbol{M}^{\left(I_{l}\right)}}\left(S^{2}\right)\left(\boldsymbol{M}^{\left(I_{l}\right)}\right.$ denotes the restriction of $\boldsymbol{M}$ to the indices of $\left.I_{l}\right)$. Recall that $A$ is irreducible, but the submatrix $A\left[I_{1} \mid I_{1}\right]$ may be reducible. So assume it is decomposable into $K_{1} \geq 1$ irreducible factors: $A\left[J_{1} \mid J_{1}\right], \ldots, A\left[J_{K_{1}} \mid J_{K_{1}}\right]$. Similarly, $A\left[I_{2} \mid I_{2}\right]$ is decomposable into $K_{2}$ irreducible factors: $A\left[\tilde{J}_{1} \mid \tilde{J}_{1}\right], \ldots, A\left[\tilde{J}_{K_{2}} \mid \tilde{J}_{K_{2}}\right]$. Fix any $1 \leq k \leq K_{1}$. Since $A$ is irreducible there exist $i_{1} \in J_{k}$ and $i_{2} \in I_{2}$ such that $a_{i_{1}, i_{2}}<0$. From 5.60) and Lemma 5.1 it follows that $\mu_{i_{1}}=M_{i_{1}} \delta_{x_{i_{1}}}$ and $\mu_{i_{2}}=M_{i_{2}} \delta_{x_{i_{2}}}$ with $x_{i_{1}}$ and $x_{i_{2}}$ a pair of antipodal points. By (5.63), the restriction of $\boldsymbol{\rho}^{(m)}$ to the indices of $\boldsymbol{J}_{k}$ is a minimizing sequence for $\Psi_{S^{2}}^{\left(J_{k}\right)}$ over $\boldsymbol{M}^{\left(J_{k}\right)}$, so that by Lemma 5.2 we deduce that $\mu_{i}=M_{i} \delta_{\widehat{x}_{k}}$ for all $i \in J_{k}$, for some $\widehat{x}_{k} \in S^{2}$. This holds for every $1 \leq k \leq K_{1}$. Similarly, $\mu_{i}=M_{i} \delta_{\widehat{y}_{k}}$ if $i \in \tilde{J}_{k}$ for $1 \leq k \leq K_{2}$.

Next we claim that $\widehat{x}_{k}=\widehat{x}$ for all $k$ and $\widehat{y}_{k}=\widehat{y}$ for all $k$, where $\widehat{x}$ and $\widehat{y}$ are antipodal, i.e. $\widehat{y}=-\widehat{x}$. We define a bipartite graph whose vertices are given by the points $\left\{\widehat{x}_{k}\right\}_{k=1}^{K_{1}}$, $\left\{\widehat{y}_{k}\right\}_{k=1}^{K_{2}}$. This graph contains an edge $\left[\widehat{x}_{k}, \widehat{y}_{l}\right]$ if and only if there exist $i \in J_{k}, j \in \tilde{J}_{l}$ with $a_{i, j} \neq 0$. We recall that if $\left[\widehat{x}_{k}, \widehat{y}_{l}\right]$ is an edge then $\widehat{x}_{k}=-\widehat{y}_{l}$. By assumption, this is a connected graph and the claim follows.

The second case that we are able to treat allows for an arbitrary number of blocks, but requires a very particular structure of the matrix $A$.

Proposition 5.2. Assume $A$ and $M$ are such that conditions $(\mathrm{P} 1)-(\mathrm{P} 2)$ are satisfied. Suppose further that there exist $n$ nonnegative numbers $\alpha_{1}, \ldots, \alpha_{n}$ and a symmetric matrix $B=\left(b_{l, m}\right)_{l, m=1}^{K}$ with nonpositive off-diagonal elements such that $a_{i, j}=\alpha_{i} \alpha_{j} b_{l, m}$ for all $j \in I_{m}, i \in I_{l}$ whenever $m \neq l$. Then

$$
\inf _{\boldsymbol{\Gamma}_{\boldsymbol{M}}\left(S^{2}\right)} \Psi_{S^{2}}=\sum_{l=1}^{K} \sum_{i \in I_{l}}\left[M_{i} \log \frac{M_{i}}{4 \pi}+\frac{M_{i}}{2 \pi} c_{0}\right]-\frac{1}{4 \pi} \sup _{\widehat{\boldsymbol{x}} \in\left(S^{2}\right)^{K}} \widehat{W}(\widehat{\boldsymbol{x}}) .
$$


If, in addition, $\widehat{A}$ does not have a row of zeros (ignoring the diagonal), then the infimum in 5.64) is not attained and any weak limit $\boldsymbol{\mu}$ of a minimizing sequence is of the form

$$
\mu_{i}=M_{i} \delta_{\widehat{x}_{l}} \quad \text { if } i \in I_{l}, 1 \leq l \leq K,
$$

where $\widehat{\boldsymbol{x}}$ is a maximizer of $\widehat{W}$.

Proof. We may rewrite 5.36 as

$$
\Psi_{S^{2}}(\boldsymbol{\rho})=\sum_{l=1}^{K} \Psi_{S^{2}}^{\left(I_{l}\right)}(\boldsymbol{\rho})+\frac{1}{4 \pi} \sum_{l \neq m} b_{l, m} \int_{S^{2}} \int_{S^{2}} \bar{\rho}_{l}(x)(\log |x-y|) \bar{\rho}_{m}(y) d x d y,
$$

where

$$
\bar{\rho}_{l}=\sum_{i \in I_{l}} \alpha_{i} \rho_{i}, \quad l=1, \ldots, K .
$$

Applying Lemma 5.1 we get

$$
\frac{1}{4 \pi} \sum_{l \neq m} b_{l, m} \int_{S^{2}} \int_{S^{2}} \bar{\rho}_{l}(x)(\log |x-y|) \bar{\rho}_{m}(y) d x d y \geq-\frac{1}{4 \pi} \sup _{\widehat{\boldsymbol{x}} \in\left(S^{2}\right)^{K}} \bar{W}(\widehat{\boldsymbol{x}}),
$$

where

$$
\bar{W}(\widehat{\boldsymbol{x}}):=\sum_{l \neq m}\left(-b_{l, m}\right) \bar{M}_{l} \bar{M}_{m} \log \left|\widehat{x}_{l}-\widehat{x}_{m}\right|
$$

and $\bar{M}_{l}:=\sum_{i \in I_{l}} \alpha_{i} M_{i}, l=1, \ldots, K$. Using the identity $\widehat{a}_{l, m} \widehat{M}_{l} \widehat{M}_{m}=b_{l, m} \bar{M}_{l} \bar{M}_{m}$ we see that $\bar{W}=\widehat{W}$. Hence, from (5.65), 5.66) and 5.59) we infer that the r.h.s. in 5.64) is a lower bound for $\Psi_{S^{2}}$. The optimality of this bound follows by considering the limit $\lim _{\alpha \rightarrow \infty} \Psi_{S^{2}}\left(\boldsymbol{\rho}^{(\alpha)}\right)$, where

$$
\rho_{i}^{(\alpha)}=\left(\rho_{i}^{0}\right)^{\tau_{\widehat{x} m, \alpha}}, \quad \forall i \in I_{m}, m=1, \ldots, K,
$$

and $\widehat{\boldsymbol{x}}=\left(\widehat{x}_{1}, \ldots, \widehat{x}_{K}\right)$ is a maximizer for $\widehat{W}$. If $\widehat{A}$ does not have a row of zeros (outside the diagonal) then the same holds for $B$. Applying Lemma 5.1 to

$$
\bar{J}(\boldsymbol{\mu})=\sum_{l \neq m}\left(-b_{l, m}\right) \int_{S^{2}} \int_{S^{2}} \log |x-y| d \mu_{l}(x) d \mu_{m}(y)
$$

over

$$
\mathcal{G}_{\bar{M}}\left(S^{2}\right)=\left\{\boldsymbol{\mu}=\left(\mu_{1}, \ldots, \mu_{K}\right): \mu_{i} \geq 0 \text { and } \int_{S^{2}} d \mu_{i}=\bar{M}_{i}, i=1, \ldots, K\right\},
$$

we deduce as in the proof of Theorem 3 (iii) the nonexistence of a minimizer and the description of the weak limits of the minimizing sequences.

Acknowledgments. Part of this work was done while the first author (I.S.) was visiting the Laboratoire J.L. Lions at the University Paris VI. He is grateful to Prof. Haim Brezis for the invitation and many interesting discussions. This research was supported by the Israel Science Foundation (grant No. 77/01). 


\section{References}

[1] Aubin, T.: Meilleures constantes dans le théorème d'inclusion de Sobolev et un théorème de Fredholm non linéaire pour la transformation conforme de la courbure scalaire. J. Funct. Anal. 32, 148-174 (1979) Zbl 0411.46019 MR 0534672

[2] Baernstein, A., Taylor, B. A.: Spherical rearrangements, subharmonic functions, and *functions in $n$-space. Duke Math. J. 43, 245-268 (1976) Zbl 0331.31002 MR 0402083

[3] Beckner, W.: Sharp Sobolev inequalities on the sphere and the Moser-Trudinger inequality. Ann. of Math. 128, 213-242 (1993) Zbl 0826.58042 MR 1230930 MR 1473855

[4] Carlen, E., Loss, M.: Competing symmetries, the logarithmic HLS inequality and Onofri's inequality on $S^{n}$. Geom. Funct. Anal. 1, 90-104 (1992) Zbl 0754.47041 MR 1143664

[5] Chang, S.-Y. A., Yang, P.: Prescribing Gaussian curvature on $S^{2}$. Acta Math. 159, 215-259 (1987) Zbl 0636.53053 MR 0908146

[6] Chanillo, S., Kiessling, M. K.-H.: Conformally invariant systems of nonlinear PDE of Liouville type. Geom. Func. Anal. 5, 924-947 (1995) Zbl 0858.35035 MR 1361515

[7] Chipot, M., Shafrir, I., Wolansky, G.: On the solutions of Liouville systems. J. Differential Equations 140, 59-105 (1997); Erratum, loc. cit. 178, 630 (2002) Zbl 0902.35039 MR 1473855

[8] Dautray, R., Lions, J.-L.: Mathematical Analysis and Numerical Methods for Science and Technology. Vol. 1, Springer, Berlin (1988) Zbl 0944.47002 MR 0918560

[9] Fontana, L.: Sharp borderline Sobolev inequalities on compact Riemannian manifolds. Comment. Math. Helv. 68, 415-454 (1993) Zbl 0844.58082 MR 1236762

[10] Hong, C.: A best constant and the Gaussian curvature. Proc. Amer. Math. Soc. 97, 737-747 (1986) Zbl 0603.58056 MR 0845999

[11] Jost, J., Wang, G.: Analytic aspects of the Toda system. I. A Moser-Trudinger inequality. Comm. Pure Appl. Math. 54, 1289-1319 (2001) Zbl pre01860529 MR 1846799

[12] Lieb, E. H.: Sharp constants in the Hardy-Littlewood-Sobolev and related inequalities. Ann. of Math. 118, 349-374 (1983) Zbl 0527.42011 MR 0717827

[13] Moser, J.: A sharp form of an inequality by N. Trudinger. Indiana Univ. Math. J. 20, 10771092 (1971) Zbl 0213.13001 MR 0301504

[14] Nolasco, M., Tarantello, G.: On a sharp Sobolev type inequality on two dimensional compact manifolds. Arch. Rat. Mech. Anal. 145, 161-195 (1998) Zbl 0980.46022 MR 1664542

[15] Onofri, E.: On the positivity of the effective action in a theory of random surfaces. Comm. Math. Phys. 86, 321-326 (1982) Zbl 0506.47031 MR 0677001

[16] Osgood, B., Phillips, R., Sarnak, P.: Extremals of determinants of Laplacians. J. Funct. Anal. 80, 148-211 (1988) Zbl 0653.53022 MR 0960228

[17] Ransford, R.: Potential Theory in the Complex Plane. London Math. Soc. Student Texts 28, Cambridge Univ. Press (1995) Zbl 0828.31001 MR 1334766

[18] Shafrir, I., Wolansky, G.: Moser-Trudinger type inequalities for systems in two dimensions. C. R. Acad. Sci. Paris Sér. I Math. 333, 439-443 (2001) Zbl pre01686428 MR 1859233

[19] Shafrir, I., Wolansky, G.: The logarithmic HLS inequality for systems on compact manifolds. J. Funct. Anal., to appear

[20] Wang, G.: Moser-Trudinger inequalities and Liouville systems. C. R. Acad. Sci. Paris Sér. I Math. 328, 895-900 (1999) MR 1689869 Zbl 0933.37064 